\title{
RATIONAL PROCESSES RELATED TO COMMUNICATING MARKOV PROCESSES
}

\author{
PETER BUCHHOLZ,* TU Dortmund \\ MIKLÓS TELEK, ${ }^{* *}$ Technical University of Budapest
}

\begin{abstract}
We define a class of stochastic processes, denoted as marked rational arrival processes (MRAPs), which is an extension of matrix exponential distributions and rational arrival processes. Continuous-time Markov processes with labeled transitions are a subclass of this more general model class. New equivalence relations between processes are defined, and it is shown that these equivalence relations are natural extensions of strong and weak lumpability and the corresponding bisimulation relations that have been defined for Markov processes. If a general rational process is equivalent to a Markov process, it can be used in numerical analysis techniques instead of the Markov process. This observation allows one to apply MRAPs like Markov processes and since the new equivalence relations are more general than lumpability and bisimulation, it is sometimes possible to find smaller representations of given processes. Finally, we show that the equivalence is preserved by the composition of MRAPs and can therefore be exploited in compositional modeling.
\end{abstract}

Keywords: Markov process; rational process; equivalence; lumpability

2010 Mathematics Subject Classification: Primary 60G20

Secondary $60 \mathrm{~J} 27$

\section{Introduction}

Markov processes in continuous time have been successfully applied for a long time in performance and dependability analysis [24]. In the last two decades compositional analysis approaches and equivalence relations based on different versions of lumpability have been proposed for Markov processes with marked transitions [5], [15], [16]. Several model classes which differ in detail but follow the same philosophies of composing communicating processes have been proposed and successfully applied.

The major advantages of Markov models are their intuitive stochastic interpretation, the possibility to compute stationary and transient results with a high precision using numerical techniques, and computable equivalence relations that allow for a reduction of the state space in several models. If one drops the first aspect, namely the stochastic interpretation, it is possible to define distributions and processes at a purely algebraic level. The first model of this kind is the class of matrix exponential (ME) distributions [14], [19], which are an extension of phase-type (PH) distributions. ME distributions have been extended to rational arrival processes (RAPs) [2], [20], which are an extension of Markovian arrival processes (MAPs) [21]. Although it has

Received 24 June 2010; revision received 15 August 2011.

* Postal address: Informatik IV, TU Dortmund, D-44221 Dortmund, Germany.

Email address: peter.buchholz@udo.edu

** Postal address: Department of Telecommunications, Technical University of Budapest, H-1521 Budapest, Hungary.

Email address: telek@hit.hme.hu 
been shown recently that ME/ME/. queues can be solved with matrix analytical methods [1], [4] and some fitting approaches have been proposed for this class of processes [12], [25], the usability of ME distributions is limited by the missing probabilistic interpretation and even more by the lack of approaches to decide whether a vector matrix pair describes an ME distribution.

Recently, we developed an equivalence relation between $\mathrm{PH}$ and ME distributions and showed in the setting of stochastic Petri nets (SPNs) that PH distributions can be substituted by equivalent ME distributions without altering marginal probabilities of the stochastic process [9]. A similar result has been proposed in [10] for MAPs and RAPs. In this paper we extend the mentioned results to a general class of stochastic processes which will be denoted as MRAPs. We formally define the class and show that it contains Markov processes with labeled transitions. Then we define two equivalence relations for MRAPs and prove that these equivalence relations are extensions of ordinary and weak lumpability or bisimulation. We can additionally prove that equivalent processes behave identically in the sense that we can substitute the matrices of one process with the matrices of another equivalent process without changing the joint densities of observing sequences of events. Consequently, if an arbitrary MRAP is equivalent to a Markov process, it can be used in stochastic models that are solved with numerical methods. This is especially attractive if MRAPs with a smaller state space that are equivalent to Markov processes can be found. It is well known that lumpability, or better bisimulation, is preserved by composition via synchronized transitions [7], [15], [16]. We show that similar results hold for our more general equivalence relations.

The paper is structured as follows. In Section 2 MRAPs are defined and their relation to ME distributions and Markov processes with marked transitions is outlined. In Section 3 the new equivalence relations are introduced; we show that they extend ordinary and weak lumpability and prove that equivalent processes can be analyzed with almost the same numerical methods. In Section 4 the composition of MRAPs is defined and we show that equivalence is preserved after composition. The paper ends with the conclusions.

\section{Rational arrival processes with multiple event types}

We begin with the definition of ME distributions and RAPs. In the following $s=\{0, \ldots$, $n-1\}$ denotes the set of states. Let $\boldsymbol{G}_{0} \in \mathbb{R}^{n, n}$ be an $n \times n$ matrix whose eigenvalues have negative real parts, which implies that $\boldsymbol{G}_{0}$ is nonsingular [19]. Furthermore, $\boldsymbol{\pi} \in \mathbb{R}^{n}$ is a vector with $\boldsymbol{\pi} \mathbf{1}=1$. Then $\left(\boldsymbol{\pi}, \boldsymbol{G}_{0}\right)$ is an ME distribution if and only if

$$
F_{\left(\pi, G_{0}\right)}(t)=1-\pi \mathrm{e}^{\boldsymbol{G}_{0} t} \mathbf{1}
$$

is a valid distribution function, where $\mathbf{1}$ is a column vector of appropriate size with all elements equal to 1 . Depending on the context, we occasionally explicitly show the sizes of the vectors, e.g. in this case $\mathbf{1}_{n}$. We assume that $F(0)=0$, which follows from $\boldsymbol{\pi} \mathbf{1}=1$. If the nondiagonal elements of $\boldsymbol{G}_{0}$ and the vector $\boldsymbol{\pi}$ are nonnegative, then (1) always describes a valid distribution and we obtain a PH distribution. For $n=2$, the classes of ME and PH distributions coincide, but, for larger dimensions, the class of ME distributions is larger [17]. On the other hand, it is known that every ME distribution with a strictly positive density on $(0, \infty)$ has a $\mathrm{PH}$ representation of a possibly larger size [3]. Consequently, only ME distributions with a density that becomes 0 in $(0, \infty)$ cannot be represented as $\mathrm{PH}$ distributions of a finite dimension. As shown in [9], ME distributions can be used like PH distributions in stochastic models, such as SPNs, and most numerical analysis techniques are still applicable. 
We now continue with RAPs as a natural extension of ME distributions to processes. Instead of the usual definition focusing only on the stationary behavior, we define the process together with an initial vector to describe its transient behavior.

Definition 1. An initial vector and a pair of matrices $\left(\boldsymbol{\pi}, \boldsymbol{G}_{0}, \boldsymbol{G}_{1}\right)$ define a rational arrival process if

1. $\boldsymbol{\pi} \mathbf{1}=1$,

2. $\left(\boldsymbol{G}_{0}+\boldsymbol{G}_{1}\right) \mathbf{1}=\mathbf{0}$,

3. all eigenvalues of $\boldsymbol{G}_{0}$ have a negative real part, which implies that the matrix is nonsingular [19],

4. $f_{\left(\boldsymbol{\pi}, \boldsymbol{G}_{0}, \boldsymbol{G}_{1}\right)}\left(t_{1}, \ldots, t_{j}\right)=\boldsymbol{\pi} \mathrm{e}^{\boldsymbol{G}_{0} t_{1}} \boldsymbol{G}_{1} \mathrm{e}^{\boldsymbol{G}_{0} t_{2}} \boldsymbol{G}_{1} \ldots \mathrm{e}^{\boldsymbol{G}_{0} t_{j}} \boldsymbol{G}_{1} \mathbf{1}$ is a valid joint density for all $t_{i} \geq 0(i=1, \ldots, j)$. That is,

$$
f_{\left(\boldsymbol{\pi}, \boldsymbol{G}_{0}, \boldsymbol{G}_{1}\right)}\left(t_{1}, \ldots, t_{j}\right) \geq 0 \text { and } \int_{t_{1}} \cdots \int_{t_{j}} f_{\left(\boldsymbol{\pi}, \boldsymbol{G}_{0}, \boldsymbol{G}_{1}\right)}\left(t_{1}, \ldots, t_{j}\right) \mathrm{d} t_{j} \cdots \mathrm{d} t_{1}=1 .
$$

If $\boldsymbol{\pi} \geq \mathbf{0}, \boldsymbol{G}_{1} \geq \mathbf{0}$, and all nondiagonal elements of $\boldsymbol{G}_{0}$ are nonnegative, then the RAP is a MAP which always describes a valid density. In contrast to MAPs, RAPs have no probabilistic interpretation at the state level. However, we can interpret the matrix $\boldsymbol{G}_{0}$ of a RAP as the origin of internal state changes and the matrix $\boldsymbol{G}_{1}$ as the origin of events or points generated by the stochastic process. The vector $\pi$ can be interpreted as the initial vector of the RAP over a set of states at time 0 . For a MAP, $\pi$ is a valid distribution; in the general case $\pi$ may contain negative elements as well. The interarrival time distribution of a RAP is an ME distribution. MAPs and RAPs can be used to model processes with a single type of event. It is natural to extend MAPs to generate multiple event types. This resulted in the definition of MMAPs [13], which can be interpreted in a more general setting as Markov processes with marked transitions. This class will be slightly extended in Section 4 to allow composition.

Similar to the extension from a MAP to an MMAP, we define a marked rational arrival process (MRAP) with $K$ event types.

Definition 2. An initial vector and a set of $K+1$ matrices $\left(\boldsymbol{\pi}, \boldsymbol{G}_{0}, \ldots, \boldsymbol{G}_{K}\right)$ define a marked rational arrival process if

1. $\boldsymbol{\pi} \mathbf{1}=1$,

2. $\left(\boldsymbol{G}_{0}+\sum_{k=1}^{K} \boldsymbol{G}_{k}\right) \mathbf{1}=\mathbf{0}$,

3. all eigenvalues of $\boldsymbol{G}_{0}$ have a negative real part, which implies that the matrix is nonsingular [19],

4.

$$
f_{\left(\boldsymbol{\pi}, \boldsymbol{G}_{0}, \ldots, \boldsymbol{G}_{K}\right)}\left(t_{1}, k_{1}, \ldots, t_{j}, k_{j}\right)=\boldsymbol{\pi} \mathrm{e}^{\boldsymbol{G}_{0} t_{1}} \boldsymbol{G}_{k_{1}} \mathrm{e}^{\boldsymbol{G}_{0} t_{2}} \boldsymbol{G}_{k_{2}} \cdots \mathrm{e}^{\boldsymbol{G}_{0} t_{j}} \boldsymbol{G}_{k_{j}} \mathbf{1}
$$

is a valid joint density for all $t_{i} \geq 0$ and $k_{i} \in\{1, \ldots, K\}(i=1, \ldots, j)$. That is,

$$
f_{\left(\pi, \boldsymbol{G}_{0}, \ldots, \boldsymbol{G}_{K}\right)}\left(t_{1}, k_{1}, \ldots, t_{j}, k_{j}\right) \geq 0
$$

and

$$
\sum_{k_{1}} \cdots \sum_{k_{j}} \int_{t_{1}} \cdots \int_{t_{j}} f_{\left(\boldsymbol{\pi}, \boldsymbol{G}_{0}, \ldots, \boldsymbol{G}_{K}\right)}\left(t_{1}, k_{1}, \ldots, t_{j}, k_{j}\right) \mathrm{d} t_{j} \cdots \mathrm{d} t_{1}=1 .
$$


As mentioned before, in these definitions the initial vector is included in the definition of MRAPs for general transient analysis. Alternatively, we can assume that the initial vector equals the embedded stationary vector $\boldsymbol{v}$ and is not part of the definition. In this case, the following condition on the matrices becomes necessary to obtain a unique stationary vector and substitutes the first condition above.

1. $\boldsymbol{P}=-\boldsymbol{G}_{0}^{-1}\left(\sum_{k=1}^{K} \boldsymbol{G}_{k}\right)$ has a unique eigenvalue 1 such that the solution $\boldsymbol{v} \boldsymbol{P}=\boldsymbol{v}, \boldsymbol{v} \mathbf{1}=1$, is unique.

Observe that the stationary vector as well as the initial vector of an MRAP may contain negative elements. The size of an MRAP equals the dimension of the vector and the matrices.

The class of MRAPs of a given size contains MMAPs of the same size since an MRAP is an MMAP if $\boldsymbol{\pi} \geq \mathbf{0}, \boldsymbol{G}_{k} \geq 0$ for $k=1, \ldots, K$, and all nondiagonal elements of $\boldsymbol{G}_{0}$ are nonnegative. MRAPs are structurally identical to BRAPs that have been defined recently in [4] to describe batch arrivals where the interarrival time process is realized by a RAP. The only difference is the interpretation of events which are not necessarily batch arrivals for MRAPs.

In general, we have to distinguish between the stochastic process and its representation. A stochastic process considered in this paper has infinitely many representations. These representations can have identical or different sizes. If we speak of an MRAP or MMAP, we always mean a representation including the vector and the set of matrices. Consequently, $\left(\boldsymbol{\pi}, \boldsymbol{G}_{0}, \ldots, \boldsymbol{G}_{K}\right)$ is an MRAP if it satifies the conditions given in Definition 2. If we mean the stochastic process then we speak of the stochastic process described by $\left(\boldsymbol{\pi}, \boldsymbol{G}_{0}, \ldots, \boldsymbol{G}_{K}\right)$. We say that a stochastic process is an MRAP process if an MRAP representation $\left(\boldsymbol{\pi}, \boldsymbol{G}_{0}, \ldots, \boldsymbol{G}_{K}\right)$ exists that describes the process. Similarly, a stochastic process is an MMAP process if it can be described by an MMAP (representation).

Consequently, in the sequel, MRAP and MMAP mean representations and if explicit reference to processes is needed, they are referred to as MRAP and MMAP processes. Implicitly, MRAP and MMAP (representations) also mean processes. Note that an MMAP process can also be described by MRAP representations. This way an MMAP representation always describes an MMAP process, but an MRAP representation can describe both MRAP and MMAP processes.

Another issue is the size of the representation. It might happen that an MMAP process has an MRAP representation of size $n$ and its smallest MMAP representation is of size $m>n$.

Example 1. The following vector and matrices represent an MMAP with two classes:

$$
\begin{gathered}
\boldsymbol{\pi}=(0.5,0,0,0.5), \quad \boldsymbol{G}_{0}=\left(\begin{array}{cccc}
-1 & 1 & 0 & 0 \\
0 & -2 & 2 & 0 \\
0 & 0 & -3 & 3 \\
0 & 0 & 0 & -4
\end{array}\right), \\
\boldsymbol{G}_{1}=\left(\begin{array}{cccc}
0 & 0 & 0 & 0 \\
0 & 0 & 0 & 0 \\
0 & 0 & 0 & 0 \\
1.5 & 0 & 0 & 1.5
\end{array}\right), \quad \text { and } \quad \boldsymbol{G}_{2}=\left(\begin{array}{cccc}
0 & 0 & 0 & 0 \\
0 & 0 & 0 & 0 \\
0 & 0 & 0 & 0 \\
0.5 & 0 & 0 & 0.5
\end{array}\right) .
\end{gathered}
$$

The following vector and matrices represent an MRAP:

$$
\boldsymbol{\phi}=(0,0,1), \quad \boldsymbol{H}_{0}=\left(\begin{array}{ccc}
-1.36364 & 4.13365 & -6.65777 \\
-1.14992 & -1.46376 & 4.02489 \\
0 & 1.1726 & -3.1726
\end{array}\right)
$$




$$
\boldsymbol{H}_{1}=\left(\begin{array}{ccc}
0 & 0 & 2.91582 \\
0 & 0 & -1.05841 \\
0 & 0 & 1.5
\end{array}\right), \quad \text { and } \quad \boldsymbol{H}_{2}=\left(\begin{array}{ccc}
0 & 0 & 0.97194 \\
0 & 0 & -0.3528 \\
0 & 0 & 0.5
\end{array}\right)
$$

It contains negative elements outside the diagonal of $\boldsymbol{H}_{0}$ and, therefore, is not an MMAP. We will later prove that these general matrices indeed define a valid stochastic process and, even more, that the MMAP and the MRAP are different representations of the same stochastic process, and, consequently an MMAP process.

\section{Equivalence relations for MRAPs}

For continuous-time Markov chains (CTMCs) with marked transitions, the concept of ordinary and exact lumpability [6], [18] provides the basis for introducing stochastic bisimulation [7], [16] that defines a concept of equivalence between different processes. CTMCs with marked transitions can be seen as a generalization of MMAPs such that lumpability and bisimulation can also be used to define equivalence between MMAPs. Bisimulation has not yet been formally defined for RAPs or MRAPs, but, as shown recently in [8], bisimulation can be extended to transitions with labels taken from an arbitrary semiring and the general proofs can be easily transferred to the specific case of RAPs showing the exact aggregation property, which means that the RAP resulting from aggregation is equivalent to the original one. Here we show that lumpability and bisimulation are special cases of a more general concept of computing equivalent representations of a minimal size. Lumpability and stochastic bisimulation are two closely related concepts. Lumpability [18] has been defined for Markov chains without marked transitions. The term bisimulation was originally used for automata models without any stochasticity [22], and has been extended to stochastic process algebras [16] and stochastic automata [7] which can be interpreted as Markov chains with marked transitions. It turns out that stochastic bisimulation is a natural extension of ordinary lumpability [6] by applying the conditions to more than one matrix. The situation is different for weak lumpability [18], [23], for which no corresponding bisimulation relation has yet been defined. In the sequel, we also mainly use the term lumpability, instead of bisimulation, for the equivalence between MRAPs. However, (ordinary) lumpability and (stochastic) bisimulation can be taken as equivalent for MMAPs and MRAPs.

We first consider lumpability and then extend it to a more general relation between processes. We then do the same for weak lumpability. Lumpability is based on a mapping between sets of states. Let $\delta=\{0, \ldots, m-1\}$ be the set of states of an MRAP $\left(\boldsymbol{\pi}, \boldsymbol{G}_{0}, \ldots, \boldsymbol{G}_{K}\right)$, and let $\hat{s}=\{0, \ldots, n-1\}, m>n$, be another set which defines a set of equivalence classes that partition $\delta$ into disjoint subsets. We denote by $[h] \subseteq \delta$ the states belonging to equivalence class $h \in \hat{\delta}$, and assume that all equivalence classes are nonempty. The mapping of states to equivalence classes can be defined by an $m \times n$ matrix $\boldsymbol{V}$ such that $\boldsymbol{V}(i, h)=1$ if $i \in[h]$ and 0 otherwise. The matrix $\boldsymbol{V}$ contains one element equal to 1 in each row (i.e. $\mathbf{1}_{m}=\boldsymbol{V} \mathbf{1}_{n}$, where $\mathbf{1}_{m}$ is a column vector of 1 s of length $m$ ) and at least one element equal to 1 in each column. The matrix $\boldsymbol{V}$ describes a lumpable partition if and only if, for all $h \in \hat{s}$ and all $i, j \in[h]$,

(C1) $\boldsymbol{e}_{i} \boldsymbol{G}_{k} \boldsymbol{V}=\boldsymbol{e}_{j} \boldsymbol{G}_{k} \boldsymbol{V}$ for all $k \in\{0, \ldots, K\}$, where $\boldsymbol{e}_{i}$ is a row vector with 1 in position $i$ and 0 elsewhere.

This definition of lumpability is a natural generalization of the definition of lumpability for Markov processes [18] and corresponds to bisimulation for Markov processes with labeled transitions [7], [16]. Observe that the matrix $\boldsymbol{V}$ has full column rank since in every column at least 
one nonzero element appears. We now extend this approach to MRAPs. Let $\left(\boldsymbol{\pi}, \boldsymbol{G}_{0}, \ldots, \boldsymbol{G}_{K}\right)$ be an MRAP with the set of states $\&$, and let $\hat{\delta}$ be a set of equivalence classes with the matrix $\boldsymbol{V}$ defining the mapping of states to equivalence classes. Assume that $\boldsymbol{V}$ describes a lumpable partition. Then a vector and a set of matrices $\left(\boldsymbol{\phi}, \boldsymbol{H}_{0}, \ldots, \boldsymbol{H}_{K}\right)$ on the set of states $\hat{s}$ can be defined as follows:

1. $\phi=\pi V$,

2. $\boldsymbol{H}_{k}(f, h)=\sum_{j \in[h]} \boldsymbol{G}_{k}(i, j)$ for some $i \in[f]$, and, hence, all $i \in[f]$ by condition (C1), and $k \in\{0, \ldots, K\}$.

We denote the computation of $\left(\boldsymbol{\phi}, \boldsymbol{H}_{0}, \ldots, \boldsymbol{H}_{K}\right)$ from $\left(\boldsymbol{\pi}, \boldsymbol{G}_{0}, \ldots, \boldsymbol{G}_{K}\right)$ as an aggregation. If $\left(\boldsymbol{\pi}, \boldsymbol{G}_{0}, \ldots, \boldsymbol{G}_{K}\right)$ is an MMAP then $\left(\boldsymbol{\phi}, \boldsymbol{H}_{0}, \ldots, \boldsymbol{H}_{K}\right)$ is also an MMAP [7], [16]. We now show that the aggregation of an MRAP is an MRAP.

Theorem 1. Let $\left(\boldsymbol{\pi}, \boldsymbol{G}_{0}, \ldots, \boldsymbol{G}_{K}\right)$ be an MRAP with a set of states 8 , and let $\boldsymbol{V}$ be a matrix describing a mapping from $\&$ into a set of equivalence classes $\hat{\delta}$. If $\boldsymbol{V}$ describes a lumpable partition (i.e. observes condition $(C 1))$ then $\left(\boldsymbol{\phi}, \boldsymbol{H}_{0}, \ldots, \boldsymbol{H}_{K}\right)$, which results from the aggregation with matrix $\boldsymbol{V}$, is an MRAP and we have $\boldsymbol{G}_{k} \boldsymbol{V}=\boldsymbol{V} \boldsymbol{H}_{k}$ for all $k \in\{0, \ldots, K\}$.

Proof. We first prove that $\boldsymbol{G}_{k} \boldsymbol{V}=\boldsymbol{V} \boldsymbol{H}_{k}$ holds if condition (C1) holds. Here $\boldsymbol{G}_{k} \boldsymbol{V}$ and $\boldsymbol{V} \boldsymbol{H}_{k}$ are $m \times n$ matrices. Element $(i, h)$ of $\boldsymbol{G}_{k} \boldsymbol{V}$ is $\sum_{j \in[h]} \boldsymbol{G}_{k}(i, j)$, which equals $\boldsymbol{H}_{k}(f, h)$ with $i \in[f]$. Since $\boldsymbol{V}(i, f)=1$ and this is the only nonzero element in row $i$, element $(i, h)$ is identical in both matrices and this holds for all $i \in \delta$ and $h \in \hat{\delta}$.

To show that $\left(\boldsymbol{\phi}, \boldsymbol{H}_{0}, \ldots, \boldsymbol{H}_{K}\right)$ is an MRAP, we have to show that the vector and matrices satisfy the four conditions for MRAPs. First, we have $\boldsymbol{\phi} \mathbf{1}_{n}=\boldsymbol{\pi} \boldsymbol{V} \mathbf{1}_{m}=\boldsymbol{\pi} \mathbf{1}_{m}=1$.

Then we have

$$
\mathbf{0}=\sum_{k=0}^{K} \boldsymbol{G}_{k} \mathbf{1}_{m}=\sum_{k=0}^{K} \boldsymbol{G}_{k} \boldsymbol{V} \mathbf{1}_{n}=\boldsymbol{V}\left(\sum_{k=0}^{K} \boldsymbol{H}_{k}\right) \mathbf{1}_{n} .
$$

Since $\boldsymbol{V}$ has full column rank (because all equivalence classes are nonempty), the equation implies that $\sum_{k=0}^{K} \boldsymbol{H}_{k} \mathbf{1}_{n}=\mathbf{0}$. To prove the third condition, let $\lambda$ be an eigenvalue of $\boldsymbol{H}_{0}$. Then $\lambda \boldsymbol{x}=\boldsymbol{H}_{0} \boldsymbol{x}$ for some nonzero vector $\boldsymbol{x}$, i.e. $\boldsymbol{x}$ is the right eigenvector belonging to eigenvalue $\lambda$. Furthermore, we have

$$
\lambda \boldsymbol{x}=\boldsymbol{H}_{0} \boldsymbol{x} \quad \Longrightarrow \quad \boldsymbol{V} \lambda \boldsymbol{x}=\boldsymbol{V} \boldsymbol{H}_{0} \boldsymbol{x} \quad \Longleftrightarrow \quad \lambda(\boldsymbol{V} \boldsymbol{x})=\boldsymbol{G}_{0}(\boldsymbol{V} \boldsymbol{x}),
$$

where $\boldsymbol{V} \boldsymbol{x}$ is nonzero due to the full column rank of $\boldsymbol{V}$. This way $\lambda$ is also an eigenvalue of $\boldsymbol{G}_{0}$ and $\boldsymbol{V} \boldsymbol{x}$ is the corresponding right eigenvector. Since all eigenvalues of $\boldsymbol{G}_{0}$ have a negative real part, the same holds for $\lambda$ and, thus, the eigenvalues of $\boldsymbol{H}_{0}$. It remains to show that $f_{\left(\boldsymbol{\phi}, \boldsymbol{H}_{0}, \ldots, \boldsymbol{H}_{K}\right)}\left(t_{1}, k_{1}, \ldots, t_{j}, k_{j}\right)$ is a valid density. This is done in a more general setting in the proof of Theorem 2 below.

Theorem 1 extends lumpability and bisimulation to MRAPs. Consequently, we can speak of an aggregated MRAP and the aggregation has a physical meaning, namely the representation of a set of states by a single state. However, it is interesting to note that the proof of Theorem 1 exploits the relations $\boldsymbol{G}_{k} \boldsymbol{V}=\boldsymbol{V} \boldsymbol{H}_{k}$ and $\mathbf{1}_{m}=\boldsymbol{V} \mathbf{1}_{n}$, and the property that $\boldsymbol{V}$ has full column rank, but does not use the fact that $\boldsymbol{V}$ describes a proper mapping, which implies that elements of $\boldsymbol{V}$ are from $\{0,1\}$. If we skip this condition, we lose the physical meaning given by the aggregation, but it is still possible to define an algebraic relation between matrices and vectors. 
This relation applied to MRAPs relates processes with an equivalent behavior as shown now. The following definition is algebraic, it relates sets of matrices and vectors of different sizes.

Definition 3. Representation $\left(\boldsymbol{\pi}, \boldsymbol{G}_{0}, \ldots, \boldsymbol{G}_{K}\right.$ ) (composed of an initial vector and the set of $K+1$ matrices) with the set of states $\delta=\{0, \ldots, m-1\}$ and representation $\left(\boldsymbol{\phi}, \boldsymbol{H}_{0}, \ldots, \boldsymbol{H}_{K}\right)$ with the set of states $\hat{s}=\{0, \ldots, n-1\}, n \leq m$, are ordinarily related if an $m \times n$ matrix $\boldsymbol{V}$ of column rank $n$ exists such that

1. $\mathbf{1}_{m}=\boldsymbol{V} \mathbf{1}_{n}$,

2. $\pi \boldsymbol{V}=\boldsymbol{\phi}$, and

3. $\boldsymbol{G}_{k} \boldsymbol{V}=\boldsymbol{V} \boldsymbol{H}_{k}$ for all $k \in\{0, \ldots, K\}$.

Definition 3 extends lumpability since matrix $\boldsymbol{V}$ now contains arbitrary elements. Consequently, $\boldsymbol{V}$ no longer defines a partition. Additionally, the above relation can relate Markov and non-Markov representations when applied to stochastic processes, as shown by the examples below. The condition that $\boldsymbol{V}$ of size $m \times n$ has full column rank can be relaxed according to Theorem 7 in Appendix A. If the rank of $\boldsymbol{V}$ is $r(r<n)$ then there exits a modified matrix $\boldsymbol{V}$ of size $m \times r$ with full column rank and an equivalent representation of size $r$.

Definition 4. Representation $\left(\boldsymbol{\pi}, \boldsymbol{G}_{0}, \ldots, \boldsymbol{G}_{K}\right)$ and representation $\left(\boldsymbol{\phi}, \boldsymbol{H}_{0}, \ldots, \boldsymbol{H}_{K}\right)$ are said to be equivalent if and only if their respective functions $f$ defined in (2) are identical, i.e.

$$
f_{\left(\boldsymbol{\pi}, \boldsymbol{G}_{0}, \ldots, \boldsymbol{G}_{K}\right)}\left(t_{1}, k_{1}, \ldots, t_{j}, k_{j}\right)=f_{\left(\boldsymbol{\phi}, \boldsymbol{H}_{0}, \ldots, \boldsymbol{H}_{K}\right)}\left(t_{1}, k_{1}, \ldots, t_{j}, k_{j}\right)
$$

for all $j \geq 0, t_{i} \geq 0$, and $k_{i} \in\{1, \ldots, K\}, i=1, \ldots, j$.

Definition 4 does not require that $\left(\boldsymbol{\pi}, \boldsymbol{G}_{0}, \ldots, \boldsymbol{G}_{K}\right)$ defines a valid MRAP process, i.e. that its $f$ function is always nonnegative. An important consequence of this equivalence definition is the following. If $\left(\boldsymbol{\pi}, \boldsymbol{G}_{0}, \ldots, \boldsymbol{G}_{K}\right)$ and $\left(\boldsymbol{\phi}, \boldsymbol{H}_{0}, \ldots, \boldsymbol{H}_{K}\right)$ are equivalent, then either $\left(\boldsymbol{\pi}, \boldsymbol{G}_{0}, \ldots, \boldsymbol{G}_{K}\right)$ and $\left(\boldsymbol{\phi}, \boldsymbol{H}_{0}, \ldots, \boldsymbol{H}_{K}\right)$ both define the same stochastic process or neither of the two representations defines a stochastic process.

Theorem 2. Representations $\left(\boldsymbol{\pi}, \boldsymbol{G}_{0}, \ldots, \boldsymbol{G}_{K}\right)$ and $\left(\boldsymbol{\phi}, \boldsymbol{H}_{0}, \ldots, \boldsymbol{H}_{K}\right)$ that are ordinarily related according to Definition 3 are equivalent.

Proof. We need to show that

$$
\boldsymbol{\pi} \mathrm{e}^{\boldsymbol{G}_{0} t_{1}} \boldsymbol{G}_{k_{1}} \mathrm{e}^{\boldsymbol{G}_{0} t_{2}} \boldsymbol{G}_{k_{2}} \cdots \mathrm{e}^{\boldsymbol{G}_{0} t_{j}} \boldsymbol{G}_{k_{j}} \mathbf{1}_{m}=\boldsymbol{\phi} \mathrm{e}^{\boldsymbol{H}_{0} t_{1}} \boldsymbol{H}_{k_{1}} \mathrm{e}^{\boldsymbol{H}_{0} t_{2}} \boldsymbol{H}_{k_{2}} \cdots \mathrm{e}^{\boldsymbol{H}_{0} t_{j}} \boldsymbol{H}_{k_{j}} \mathbf{1}_{n}
$$

for all $j \geq 0, t_{i} \geq 0$, and $k_{i} \in\{1, \ldots, K\}, i=1, \ldots, j$. We prove the theorem by induction. By definition, we have, for $j=0, \boldsymbol{\pi} \boldsymbol{V}=\boldsymbol{\phi}$ such that $\boldsymbol{\pi} \mathbf{1}_{m}=\boldsymbol{\pi} \boldsymbol{V} \mathbf{1}_{n}=\boldsymbol{\phi} \mathbf{1}_{n}=1$. Let

$$
\boldsymbol{\pi}^{(i)}=\boldsymbol{\pi} \mathrm{e}^{\boldsymbol{G}_{0} t_{1}} \boldsymbol{G}_{k_{1}} \ldots \mathrm{e}^{\boldsymbol{G}_{0} t_{i}} \boldsymbol{G}_{k_{i}} \quad \text { and } \quad \boldsymbol{\phi}^{(i)}=\boldsymbol{\phi} \mathrm{e}^{\boldsymbol{H}_{0} t_{1}} \boldsymbol{H}_{k_{1}} \cdots \mathrm{e}^{\boldsymbol{H}_{0} t_{i}} \boldsymbol{H}_{k_{i}}
$$

for $i<j$. Assume that $\boldsymbol{\pi}^{(i)} \boldsymbol{V}=\boldsymbol{\phi}^{(i)}$. Then

$$
\begin{aligned}
\boldsymbol{\phi}^{(i+1)} & =\boldsymbol{\phi}^{(i)} \mathrm{e}^{\boldsymbol{H}_{0} t_{i}} \boldsymbol{H}_{k_{i}} \\
& =\boldsymbol{\phi}^{(i)} \sum_{l=0}^{\infty} \frac{\left(\boldsymbol{H}_{0} t_{i}\right)^{l}}{l !} \boldsymbol{H}_{k_{i}} \\
& =\boldsymbol{\pi}^{(i)} \boldsymbol{V} \sum_{l=0}^{\infty} \frac{\left(\boldsymbol{H}_{0} t_{i}\right)^{l}}{l !} \boldsymbol{H}_{k_{i}}
\end{aligned}
$$




$$
\begin{aligned}
& =\boldsymbol{\pi}^{(i)} \sum_{l=0}^{\infty} \frac{\left(\boldsymbol{G}_{0} t_{i}\right)^{l}}{l !} \boldsymbol{G}_{k_{i}} \boldsymbol{V} \\
& =\boldsymbol{\pi}^{(i+1)} \boldsymbol{V} .
\end{aligned}
$$

Since the relation holds for $i=0$, it holds for all $i$ and $t_{i} \geq 0$. Furthermore, $\boldsymbol{\phi}^{(i)} \mathbf{1}_{n}=$ $\boldsymbol{\pi}^{(i)} \boldsymbol{V} \mathbf{1}_{n}=\boldsymbol{\pi}^{(i)} \mathbf{1}_{m}$.

Theorem 2 relates different representations and, of course, these representations can also be MMAP and MRAP representations. Consequently, Theorem 2 can be used to prove that $\left(\boldsymbol{\phi}, \boldsymbol{H}_{0}, \ldots, \boldsymbol{H}_{K}\right)$ is a valid MRAP using the following corollary. This is particularly useful if MMAPs and MRAPs are related since an MMAP is per se a stochastic process, whereas, for general matrices, it has to be explicitly proved that the density remains nonnegative.

Corollary 1. If ( $\left.\boldsymbol{\pi}, \boldsymbol{G}_{0}, \ldots, \boldsymbol{G}_{K}\right)$ is an MRAP with $m$ states, and $\left(\boldsymbol{\phi}, \boldsymbol{H}_{0}, \ldots, \boldsymbol{H}_{K}\right)$ is a vector and a set of matrices of dimension $n(\leq m)$ which is ordinarily related to $\left(\boldsymbol{\pi}, \boldsymbol{G}_{0}, \ldots, \boldsymbol{G}_{K}\right)$ using matrix $\boldsymbol{V}$ with $\operatorname{rank}(\boldsymbol{V})=n$, then $\left(\boldsymbol{\phi}, \boldsymbol{H}_{0}, \ldots, \boldsymbol{H}_{K}\right)$ and $\left(\boldsymbol{\pi}, \boldsymbol{G}_{0}, \ldots, \boldsymbol{G}_{K}\right)$ are equivalent processes.

The relation between ordinarily related MRAPs goes beyond the equivalence of the joint densities. As shown in the proof of Theorem 2, the conditional distribution after a sequence of events of the MRAP with the larger set of states determines the conditional distribution of the MRAP with the smaller set of states $\left(\boldsymbol{\pi}^{(i)} \boldsymbol{V}=\boldsymbol{\phi}^{(i)}\right.$ ), but not vice versa (since $\boldsymbol{V}$ is not invertible).

Apart from ordinary lumpability, weak lumpability has also been defined [18, Chapter 6.4] for Markov processes. Like ordinary lumpability, it is based on a partition of the state space, but, in contrast to ordinary lumpability, it depends on the initial vector. We first define weak lumpability for MMAPs and MRAPs, which to the best of the authors' knowledge has not yet been done. Only a restricted form of weak lumpability has been defined for stochastic automata [7].

Let $\left(\boldsymbol{\pi}, \boldsymbol{G}_{0}, \ldots, \boldsymbol{G}_{K}\right)$ be an MRAP, and let $\boldsymbol{V}$ be an $m \times n(m>n)$ partition matrix. According to [18, Chapter 6.3] we define an $n \times m$ matrix

$$
\boldsymbol{W}=\left(\operatorname{diag}\left((\operatorname{diag}(\boldsymbol{\pi}) \boldsymbol{V})^{\top} \mathbf{1}_{m}\right)\right)^{-1}(\operatorname{diag}(\boldsymbol{\pi}) \boldsymbol{V})^{\top},
$$

where $\operatorname{diag}(\pi)$ is a diagonal matrix with $\pi(i)$ in position $(i, i)$ such that

$$
\boldsymbol{W}(h, i)=\boldsymbol{V}(i, h) \frac{\boldsymbol{\pi}(i)}{\sum_{l \in[h]} \boldsymbol{\pi}(l)} .
$$

We assume that $\sum_{l \in[h]} \pi(l) \neq 0$ for all $h \in \hat{s}$ to obtain a valid matrix $\boldsymbol{W}$. The matrix $\boldsymbol{W}$ has row sum 1, one nonzero element in every column, and full row rank. Furthermore, $\boldsymbol{W} \boldsymbol{V}=\boldsymbol{I}_{n}$. Let $\boldsymbol{H}_{k}=\boldsymbol{W} \boldsymbol{G}_{k} \boldsymbol{V}$ for $k=0, \ldots, K$. A necessary condition for weak lumpability is

$$
\boldsymbol{H}_{k} \boldsymbol{H}_{l}=\boldsymbol{W} \boldsymbol{G}_{k} \boldsymbol{G}_{l} \boldsymbol{V}
$$

for all $k, l \in\{0, \ldots, K\}$ [18, p. 135]. Relation (4) holds if $\boldsymbol{V}$ is a partition matrix of an ordinarily lumpable partition since then $\boldsymbol{G}_{k} \boldsymbol{V}=\boldsymbol{V} \boldsymbol{H}_{k}$ for all $k \in\{0, \ldots, K\}$ and

$$
\boldsymbol{W} \boldsymbol{G}_{k} \boldsymbol{G}_{l} \boldsymbol{V}=\boldsymbol{W} \boldsymbol{G}_{k} \boldsymbol{V} \boldsymbol{H}_{l}=\boldsymbol{H}_{k} \boldsymbol{H}_{l} .
$$


In this case, the initial vector $\pi$ is not needed for (4), since the initial vector of the MRAP with the smaller state space can always be computed as $\phi=\pi \boldsymbol{V}$ and $\boldsymbol{W}$ can be computed from $\boldsymbol{V}$ as in (3) using an arbitrary vector $\boldsymbol{\eta}>\mathbf{0}$.

A second relation for which (4) holds is $\boldsymbol{W}_{k}=\boldsymbol{H}_{k} \boldsymbol{W}$ for all $k \in\{1, \ldots, K\}$, which is defined as weak lumpability. In this case the equivalence depends on $\boldsymbol{W}$ and, therefore, also on $\pi$. Before we prove the equivalence of weakly lumpable MRAPs, we extend the equivalence as we did for ordinary lumpability and begin again with an algebraic relation between sets of matrices and vectors.

Definition 5. Representation $\left(\boldsymbol{\pi}, \boldsymbol{G}_{0}, \ldots, \boldsymbol{G}_{K}\right)$ with the set of states $\&=\{0, \ldots, m-1\}$ and representation $\left(\boldsymbol{\phi}, \boldsymbol{H}_{0}, \ldots, \boldsymbol{H}_{K}\right)$ with the set of states $\hat{\jmath}=\{0, \ldots, n-1\}, n \leq m$, are weakly related if an $n \times m$ matrix $\boldsymbol{W}$ of full row rank exists such that

1. $\mathbf{1}_{n}=\boldsymbol{W} \mathbf{1}_{m}$,

2. $\pi=\phi W$, and

3. $\boldsymbol{W} \boldsymbol{G}_{k}=\boldsymbol{H}_{k} \boldsymbol{W}$ for all $k \in\{0, \ldots, K\}$.

In contrast to weak lumpability, more general matrices $\boldsymbol{W}$ are now allowed that may contain negative elements or more than one nonzero element per column, since $\pi$ needs not be nonnegative and $\boldsymbol{V}$ needs not be a partition matrix. Again, the condition on the full row rank can be relaxed according to Theorem 8 in Appendix B.

Theorem 3. Representations $\left(\boldsymbol{\pi}, \boldsymbol{G}_{0}, \ldots, \boldsymbol{G}_{K}\right)$ and $\left(\boldsymbol{\phi}, \boldsymbol{H}_{0}, \ldots, \boldsymbol{H}_{K}\right)$ that are weakly related according to Definition 5 are equivalent.

Proof. We need to show that

$$
\boldsymbol{\pi} \mathrm{e}^{\boldsymbol{G}_{0} t_{1}} \boldsymbol{G}_{k_{1}} \mathrm{e}^{\boldsymbol{G}_{0} t_{2}} \boldsymbol{G}_{k_{2}} \cdots \mathrm{e}^{\boldsymbol{G}_{0} t_{j}} \boldsymbol{G}_{k_{j}} \mathbf{1}_{m}=\boldsymbol{\phi} \mathrm{e}^{\boldsymbol{H}_{0} t_{1}} \boldsymbol{H}_{k_{1}} \mathrm{e}^{\boldsymbol{H}_{0} t_{2}} \boldsymbol{H}_{k_{2}} \cdots \mathrm{e}^{\boldsymbol{H}_{0} t_{j}} \boldsymbol{H}_{k_{j}} \mathbf{1}_{n}
$$

for all $j \geq 0, k_{i} \in\{1, \ldots, K\}$, and $t_{i} \geq 0,0<i \leq j$. We prove the theorem by induction. By definition, we have, for $j=0, \pi=\phi \boldsymbol{W}$ such that $\boldsymbol{\phi} \mathbf{1}_{n}=\boldsymbol{\phi} \boldsymbol{W} \mathbf{1}_{m}=\boldsymbol{\pi} \mathbf{1}_{m}=1$. Define $\boldsymbol{\pi}^{(i)}$ and $\boldsymbol{\phi}^{(i)}$ as in the proof of Theorem 2, and assume that $\boldsymbol{\pi}^{(i)}=\boldsymbol{\phi}^{(i)} \boldsymbol{W}$. Then

$$
\begin{aligned}
\boldsymbol{\pi}^{(i+1)} & =\boldsymbol{\pi}^{(i)} \sum_{l=0}^{\infty} \frac{\left(\boldsymbol{G}_{0} t_{i}\right)^{l}}{l !} \boldsymbol{G}_{k_{i}} \\
& =\boldsymbol{\phi}^{(i)} \boldsymbol{W} \sum_{l=0}^{\infty} \frac{\left(\boldsymbol{G}_{0} t_{i}\right)^{l}}{l !} \boldsymbol{G}_{k_{i}} \\
& =\boldsymbol{\phi}^{(i)} \sum_{l=0}^{\infty} \frac{\left(\boldsymbol{H}_{0} t_{i}\right)^{l}}{l !} \boldsymbol{H}_{k_{i}} \boldsymbol{W} \\
& =\boldsymbol{\phi}^{(i+1)} \boldsymbol{W} .
\end{aligned}
$$

Since the relation holds for $i=0$, it holds for all $i, k_{i} \in\{1, \ldots, K\}$, and $t_{i} \geq 0$. Furthermore, $\boldsymbol{\pi}^{(i)} \mathbf{1}_{m}=\boldsymbol{\phi}^{(i)} \boldsymbol{W} \mathbf{1}_{m}=\boldsymbol{\phi}^{(i)} \mathbf{1}_{n}$.

Theorem 3 shows that an MRAP can be substituted by a smaller weakly equivalent representation since both represent the same stochastic process. The relation between weakly equivalent MRAPs goes beyond the equivalence of the joint densities, since the vector after 
observing $i$ events in the process with the smaller state space can be used to recreate the vector in the process with the larger state space. Again, the corollary below shows how to use the equivalence to prove that a representation describes a valid MRAP.

Corollary 2. If $\left(\boldsymbol{\pi}, \boldsymbol{G}_{0}, \ldots, \boldsymbol{G}_{K}\right)$ is an MRAP with $m$ states, and $\left(\boldsymbol{\phi}, \boldsymbol{H}_{0}, \ldots, \boldsymbol{H}_{K}\right)$ is a vector and a set of matrices of dimension $n(\leq m)$ which is weakly related to $\left(\boldsymbol{\pi}, \boldsymbol{G}_{0}, \ldots, \boldsymbol{G}_{K}\right)$ using matrix $\boldsymbol{W}(\operatorname{rank}(\boldsymbol{W})=n)$, then $\left(\boldsymbol{\phi}, \boldsymbol{H}_{0}, \ldots, \boldsymbol{H}_{K}\right)$ and $\left(\boldsymbol{\pi}, \boldsymbol{G}_{0}, \ldots, \boldsymbol{G}_{K}\right)$ are equivalent processes.

In the following we give a few examples of equivalent processes. In general, for an MRAP, it is possible to compute ( $\boldsymbol{\pi}, \boldsymbol{G}_{0}, \ldots, \boldsymbol{G}_{K}$ ) ordinarily or weakly equivalent representations with a minimal number of states. A preliminary version of such a reduction algorithm is presented in [10], where results from the computation of minimal representations in linear system theory [11] were used. However, a detailed description of the algorithmic reduction is beyond the scope of this paper, and so we instead consider in the following section the relation between the equivalence and composition of MRAPs.

Example 2. We consider the following MMAP of size 4:

$$
\begin{gathered}
\boldsymbol{\eta}=(0.5,0.25,0.1,0.15), \quad \boldsymbol{D}_{0}=\left(\begin{array}{ccccc}
-6 & 1.123611 & 1 & 0.3922609 & 0.1190476 \\
0 & -5 & 1.1904762 & 0.7619048 \\
0 & 3.1111111 & -6.00 & 0 \\
0 & 1.8472211 & 1.375 & -5
\end{array}\right), \\
\boldsymbol{D}_{1}=\left(\begin{array}{llll}
0 & 0 & 0.2 & 0.8 \\
0 & 0 & 0.2 & 0.8 \\
0 & 0 & 0.2 & 0.8 \\
0 & 0 & 0.2 & 0.8
\end{array}\right), \quad \text { and } \quad \boldsymbol{D}_{2}=\left(\begin{array}{cccc}
0 & 0 & 0.7 & 2.6650794 \\
0 & 0 & 0.28 & 1.7676191 \\
0 & 0 & 0.68 & 1.2088889 \\
0 & 0 & 0.08 & 0.6977778
\end{array}\right)
\end{gathered}
$$

With

$$
\boldsymbol{W}=\left(\begin{array}{cccc}
1 & 0.5 & 0 & -0.5 \\
0 & 0.7 & 0.3 & 0 \\
0 & 0 & 0.2 & 0.8
\end{array}\right)
$$

we obtain the MMAP

$$
\begin{gathered}
\boldsymbol{\pi}=(0.5,0,0.5), \quad \boldsymbol{G}_{0}=\left(\begin{array}{ccc}
-6 & 1 & 0 \\
0 & -3.66667 & 0.66667 \\
0 & 3 & -5
\end{array}\right), \\
\boldsymbol{G}_{1}=\left(\begin{array}{lll}
0 & 0 & 1 \\
0 & 0 & 1 \\
0 & 0 & 1
\end{array}\right), \quad \text { and } \quad \boldsymbol{G}_{2}=\left(\begin{array}{lll}
0 & 0 & 4 \\
0 & 0 & 2 \\
0 & 0 & 1
\end{array}\right)
\end{gathered}
$$

such that $\boldsymbol{\eta}=\boldsymbol{\pi} \boldsymbol{W}$ and $\boldsymbol{W} \boldsymbol{D}_{i}=\boldsymbol{G}_{i} \boldsymbol{W}, i=0,1,2$, which implies that they are equivalent processes according to Theorem 3 . Now define

$$
\boldsymbol{V}=\left(\begin{array}{cc}
1 & 0 \\
0.33333 & 0.66667 \\
0 & 1
\end{array}\right)
$$


Then

$$
\begin{gathered}
\boldsymbol{\phi}=(0.5,0.5), \quad \boldsymbol{H}_{0}=\left(\begin{array}{cc}
-5.66667 & 0.66667 \\
1 & -3
\end{array}\right), \\
\boldsymbol{H}_{1}=\left(\begin{array}{ll}
0 & 1 \\
0 & 1
\end{array}\right), \quad \text { and } \quad \boldsymbol{H}_{2}=\left(\begin{array}{ll}
0 & 4 \\
0 & 1
\end{array}\right)
\end{gathered}
$$

are such that $\boldsymbol{\pi} \boldsymbol{V}=\boldsymbol{\phi}$ and $\boldsymbol{G}_{i} \boldsymbol{V}=\boldsymbol{V} \boldsymbol{H}_{i}, i=0,1,2$, which implies that they are equivalent processes. Observe that neither $\boldsymbol{V}$ nor $\boldsymbol{W}$ describe a (weakly) lumpable partition.

In the example above, the two MMAPs and the final MRAP are related. It is also possible and often necessary to leave the restricted class of MMAPs to find an equivalent representation with less states. The MMAP and the MRAP shown in Example 1 are weakly related by the matrix

$$
\boldsymbol{W}=\left(\begin{array}{cccc}
1.37646 & -0.60678 & -0.74162 & 0.97194 \\
0.9264 & 0.4264 & 0 & -0.3528 \\
0.5 & 0 & 0 & 0.5
\end{array}\right)
$$

and are therefore equivalent processes.

\section{Composition of MRAPs}

MRAPs as introduced in the previous sections generate events of different types; to make them compositional, these events have to be accepted by other MRAPs, which requires an extension of the model class. We consider here asynchronous composition, which implies that we distinguish between outgoing (active) events and incoming (passive) events. This viewpoint corresponds to queueing networks without blocking [5] or probabilistic I/O automata [26]. We consider the composition of two MRAPs, and define a symmetric composition, which means that each of the MRAPs is able to send events to the other one. This is a general interpretation of an MRAP which goes beyond the interpretation as a traffic source. An MRAP is seen as a stochastic process that interacts with its environment by sending and receiving events. In this way MRAPs can be used to model interacting systems, such as processes of a distributed system that exchange messages or multiclass queueing networks where customers travel between subsystems, each described as an MRAP.

As an extension of MRAPs, we define an extended marked rational arrival process (EMRAP) with $K$ outgoing and $L$ incoming event types by an initial vector $\pi$ and a set of $K+L+1$ matrices $\left(\boldsymbol{\pi}, \boldsymbol{G}_{0}, \ldots, \boldsymbol{G}_{K}, \boldsymbol{U}_{1}, \ldots, \boldsymbol{U}_{L}\right)$ such that

1. $\left(\boldsymbol{\pi}, \boldsymbol{G}_{0}, \ldots, \boldsymbol{G}_{K}\right)$ is an MRAP,

2. $\boldsymbol{U}_{l} \mathbf{1}=\mathbf{1}$ for $l=1, \ldots, L$, and

3. $g_{\left(\boldsymbol{\pi}, \boldsymbol{G}_{0}, \ldots, \boldsymbol{G}_{K}, \boldsymbol{U}_{1}, \ldots, \boldsymbol{U}_{L}\right)}\left(t_{1}, k_{1}, \ldots, t_{j}, k_{j}\right)=\boldsymbol{v e}^{\boldsymbol{G}_{0} t_{1}} \boldsymbol{X}_{k_{1}} \mathrm{e}^{\boldsymbol{G}_{0} t_{2}} \boldsymbol{X}_{k_{2}} \ldots \mathrm{e}^{\boldsymbol{G}_{0} t_{j}} \boldsymbol{X}_{k_{j}} \mathbf{1} \geq 0$ for all $j \geq 0, t_{i} \geq 0$, and $\boldsymbol{X}_{k_{i}} \in\left\{\boldsymbol{G}_{1}, \ldots, \boldsymbol{G}_{K}, \boldsymbol{U}_{1}, \ldots, \boldsymbol{U}_{L}\right\}, i=1, \ldots, j$.

We use a general function $g$ rather than a probability density function $f$ because incoming events are included and these events are triggered by some other process. Therefore, $g$ is in general not a density, but it has to be nonnegative. If $\left(\boldsymbol{\pi}, \boldsymbol{G}_{0}, \ldots, \boldsymbol{G}_{K}\right)$ is an MMAP and $\boldsymbol{U}_{l} \geq 0$, then the resulting process is Markovian and the third condition is always observed.

The composition of two EMRAPs, $\left(\boldsymbol{\pi}^{(1)}, \boldsymbol{G}_{0}^{(1)}, \ldots, \boldsymbol{G}_{K}^{(1)}, \boldsymbol{U}_{1}^{(1)}, \ldots, \boldsymbol{U}_{L}^{(1)}\right)$ of dimension $n_{1}$ and $\left(\boldsymbol{\pi}^{(2)}, \boldsymbol{G}_{0}^{(2)}, \ldots, \boldsymbol{G}_{L}^{(2)}, \boldsymbol{U}_{1}^{(2)}, \ldots, \boldsymbol{U}_{K}^{(2)}\right)$ of dimension $n_{2}$, is defined by the following vector 
and matrices:

$$
\begin{gathered}
\boldsymbol{\pi}^{(0)}=\boldsymbol{\pi}^{(1)} \otimes \boldsymbol{\pi}^{(2)}, \\
\boldsymbol{G}_{0}^{(0)}=\boldsymbol{G}_{0}^{(1)} \oplus \boldsymbol{G}_{0}^{(2)}=\boldsymbol{G}_{0}^{(1)} \otimes \boldsymbol{I}_{n_{2}}+\boldsymbol{I}_{n_{1}} \otimes \boldsymbol{G}_{0}^{(2)}, \\
\boldsymbol{G}_{k}^{(0)}= \begin{cases}\boldsymbol{G}_{k}^{(1)} \otimes \boldsymbol{U}_{k}^{(2)} & \text { if } 1 \leq k \leq K, \\
\boldsymbol{U}_{k-K}^{(1)} \otimes \boldsymbol{G}_{k-K}^{(2)} & \text { if } K<k \leq K+L .\end{cases}
\end{gathered}
$$

Theorem 4. $\left(\boldsymbol{\pi}^{(0)}, \boldsymbol{G}_{0}^{(0)}, \ldots, \boldsymbol{G}_{K+L}^{(0)}\right)$ is an MRAP.

Proof. Let $n_{0}=n_{1} n_{2}$. We have

$$
\boldsymbol{\pi}^{(0)} \mathbf{1}=\left(\boldsymbol{\pi}^{(1)} \otimes \boldsymbol{\pi}^{(2)}\right) \mathbf{1}=\boldsymbol{\pi}^{(1)} \mathbf{1} \cdot \boldsymbol{\pi}^{(2)} \mathbf{1}=1
$$

and

$$
\begin{aligned}
\left(\boldsymbol{G}_{0}^{(0)}+\sum_{k=1}^{K+L} \boldsymbol{G}_{k}^{(0)}\right) \mathbf{1}= & \left(\boldsymbol{G}_{0}^{(1)} \otimes \boldsymbol{I}_{n_{2}}+\boldsymbol{I}_{n_{1}} \otimes \boldsymbol{G}_{0}^{(2)}+\sum_{k=1}^{K} \boldsymbol{G}_{k}^{(1)} \otimes \boldsymbol{U}_{k}^{(2)}\right. \\
& \left.+\sum_{l=1}^{L} \boldsymbol{U}_{l}^{(1)} \otimes \boldsymbol{G}_{l}^{(2)}\right) \mathbf{1} \\
= & \sum_{k=0}^{K} \boldsymbol{G}_{k}^{(1)} \mathbf{1}_{n_{1}} \otimes \mathbf{1}_{n_{2}}+\mathbf{1}_{n_{1}} \otimes \sum_{l=0}^{L} \boldsymbol{G}_{l}^{(2)} \mathbf{1}_{n_{2}} \\
= & \mathbf{0} .
\end{aligned}
$$

Furthermore, if $\mu_{1}$ and $\mu_{2}$ are eigenvalues of $\boldsymbol{G}_{0}^{(1)}$ and $\boldsymbol{G}_{0}^{(2)}$, then $\mu_{1}+\mu_{2}$ is an eigenvalue of $\boldsymbol{G}_{0}^{(0)}$, which implies that all eigenvalues of $\boldsymbol{G}_{0}^{(0)}$ have a negative real part. It remains to show that $f_{\left(\boldsymbol{\pi}^{(0)}, \boldsymbol{G}_{0}^{(0)}, \ldots, \boldsymbol{G}_{K+L}^{(0)}\right)}\left(t_{1}, k_{1}, \ldots, t_{j}, k_{j}\right)$ is a valid density. We have

$$
\mathrm{e}^{\boldsymbol{G}_{0}^{(0)} t}=\mathrm{e}^{\boldsymbol{G}_{0}^{(1)} \oplus \boldsymbol{G}_{0}^{(2)} t}=\mathrm{e}^{\boldsymbol{G}_{0}^{(1)} t} \otimes \mathrm{e}^{\boldsymbol{G}_{0}^{(2)} t},
$$

since

$$
\begin{aligned}
\mathrm{e}^{\boldsymbol{G}_{0}^{(1)} \oplus \boldsymbol{G}_{0}^{(2)} t} & =\sum_{h=0}^{\infty} \frac{\left(\left(\boldsymbol{G}_{0}^{(1)} \oplus \boldsymbol{G}_{0}^{(2)}\right) t\right)^{h}}{h !} \\
& =\sum_{h=0}^{\infty} \frac{\left(\left(\boldsymbol{G}_{0}^{(1)} \otimes \boldsymbol{I}_{n_{2}}+\boldsymbol{I}_{n_{2}} \otimes \boldsymbol{G}_{0}^{(2)}\right) t\right)^{h}}{h !} \\
& =\sum_{h=0}^{\infty} \frac{1}{h !} \sum_{i=0}^{h}\left(\begin{array}{l}
h \\
i
\end{array}\right)\left(t \boldsymbol{G}_{0}^{(1)}\right)^{i} \otimes\left(t \boldsymbol{G}_{0}^{(2)}\right)^{h-i} \\
& =\sum_{h=0}^{\infty} \frac{\left(t \boldsymbol{G}_{0}^{(1)}\right)^{h}}{h !} \otimes \sum_{i=0}^{\infty} \frac{\left(t \boldsymbol{G}_{0}^{(1)}\right)^{i}}{i !} \\
& =\mathrm{e}^{\boldsymbol{G}_{0}^{(1)} t} \otimes \mathrm{e}^{(2)} t
\end{aligned}
$$

Define $\boldsymbol{\pi}_{0}^{(i)}=\boldsymbol{\pi}^{(i)}, i \in\{0,1,2\}$, as the initial vector of the three processes. Then $\boldsymbol{\pi}_{0}^{(0)}=$ $\boldsymbol{\pi}_{0}^{(1)} \otimes \pi_{0}^{(2)}$ and, for some sequence, $\left(t_{1}, k_{1}, t_{2}, k_{2}, \ldots, t_{j}, k_{j}\right), \boldsymbol{\pi}_{j}^{(i)}=\boldsymbol{\pi}_{j-1}^{(i)} \mathrm{e}^{\boldsymbol{G}_{0}^{(i)} t_{j}} \boldsymbol{X}_{k_{j}}^{(i)}$. 
Now assume that $\pi_{j-1}^{(0)}=\pi_{j-1}^{(1)} \otimes \pi_{j-1}^{(2)}$. Then it follows by induction that, for all $j>0$,

$$
\begin{aligned}
\boldsymbol{\pi}_{j}^{(0)} & =\left(\boldsymbol{\pi}_{j-1}^{(1)} \otimes \boldsymbol{\pi}_{j-1}^{(2)}\right)\left(\mathrm{e}^{\boldsymbol{G}_{0}^{(1)} t_{j}} \otimes \mathrm{e}^{\boldsymbol{G}_{0}^{(2)} t_{j}}\right)\left(\boldsymbol{X}_{k_{j}}^{(1)} \otimes \boldsymbol{X}_{k_{j}}^{(2)}\right) \\
& =\left(\boldsymbol{\pi}_{j-1}^{(1)} \mathrm{e}^{\boldsymbol{G}_{0}^{(1)} t_{j}} \boldsymbol{X}_{k_{j}}^{(1)}\right) \otimes\left(\boldsymbol{\pi}_{j-1}^{(2)} \mathrm{e}^{\boldsymbol{G}_{0}^{(2)} t_{j}} \boldsymbol{X}_{k_{j}}^{(2)}\right) \\
& =\boldsymbol{\pi}_{j}^{(1)} \otimes \boldsymbol{\pi}_{j}^{(2)},
\end{aligned}
$$

where $\boldsymbol{X}_{k}^{(1)}=\boldsymbol{G}_{k}^{(1)}$ for $k \leq K$ and $\boldsymbol{U}_{k-K}^{(1)}$ otherwise, $\boldsymbol{X}_{k}^{(2)}=\boldsymbol{U}_{k}^{(2)}$ for $k \leq K$ and $\boldsymbol{G}_{k-K}^{(2)}$ otherwise, and $\boldsymbol{X}_{k}^{(0)}=\boldsymbol{G}_{k}^{(0)}$. Furthermore, $\boldsymbol{\pi}_{j}^{(l)} \mathbf{1}=\boldsymbol{\pi}^{(l)} \mathrm{e}^{\boldsymbol{G}_{0}^{(l)} t_{1}} \boldsymbol{X}_{k_{1}}^{(l)} \cdots \mathrm{e}^{\boldsymbol{G}_{0}^{(l)} t_{j}} \boldsymbol{X}_{k_{j}}^{(l)} \mathbf{1} \geq 0$ for $l=1,2$ and $\boldsymbol{X}_{k_{i}}^{l}$ as above since $\left(\boldsymbol{\pi}^{(l)}, \boldsymbol{G}_{0}^{(l)}, \ldots, \boldsymbol{G}_{K}^{(l)}, \boldsymbol{U}_{1}^{(l)}, \ldots, \boldsymbol{U}_{L}^{(l)}\right)$ is an EMRAP with a nonnegative function $g$. This implies that $\boldsymbol{\pi}_{j}^{(0)} \mathbf{1}=\boldsymbol{\pi}_{j}^{(1)} \mathbf{1} \cdot \boldsymbol{\pi}_{j}^{(2)} \mathbf{1} \geq 0$ for all $t$. Then $f$ is a valid density if and only if

$$
\int_{t=0}^{\infty} \sum_{k=1}^{K+L} \boldsymbol{\pi}_{j-1}^{(0)} \mathrm{e}^{\boldsymbol{G}_{0}^{(0)} t} \boldsymbol{G}_{k}^{(0)} \mathbf{1} \mathrm{d} t=\boldsymbol{\pi}_{j}^{(0)} \mathbf{1} .
$$

We have $\boldsymbol{G}_{0}^{(0)} \mathbf{1}=-\sum_{k=1}^{K+L} \boldsymbol{G}_{k}^{(0)} \mathbf{1}$ and $\lim _{t \rightarrow \infty} \mathrm{e}^{\boldsymbol{G}_{0}^{(0)} t}=\mathbf{0}$ since all eigenvalues of $\boldsymbol{G}_{0}^{(0)}$ have a negative real part such that

$$
\begin{aligned}
\int_{t=0}^{\infty} \sum_{k=1}^{K+L} \boldsymbol{\pi}_{j}^{(0)} \mathrm{e}^{\boldsymbol{G}_{0}^{(0)} t} \boldsymbol{G}_{k}^{(0)} \mathbf{1} \mathrm{d} t & =\boldsymbol{\pi}_{j}^{(0)} \int_{t=0}^{\infty} \mathrm{e}^{\boldsymbol{G}_{0}^{(0)} t} \mathrm{~d} t \sum_{k=1}^{K+L} \boldsymbol{G}_{k}^{(0)} \mathbf{1} \\
& =\boldsymbol{\pi}_{j}^{(0)}\left(\boldsymbol{G}_{0}^{(0)}\right)^{-1}(\mathbf{0}-\boldsymbol{I})\left(-\boldsymbol{G}_{0}^{(0)}\right) \mathbf{1} \\
& =\boldsymbol{\pi}_{j}^{(0)} \mathbf{1} .
\end{aligned}
$$

It is easy to show that the composition of two EMRAPs which are Markovian results in an MMAP.

\section{Preservation of equivalence after composition}

We now show that equivalence is preserved by composition such that equivalent EMRAPs can be substituted into a composition and the result is an equivalent composed MRAP. Before this can be shown, equivalence has to be defined for EMRAPs by extending the definitions for MRAPs.

Definition 6. Two EMRAPs, $\left(\boldsymbol{\pi}, \boldsymbol{G}_{0}, \ldots, \boldsymbol{G}_{K}, \boldsymbol{U}_{1}, \ldots, \boldsymbol{U}_{L}\right)$ of size $m$ and $\left(\boldsymbol{\phi}, \boldsymbol{H}_{0}, \ldots, \boldsymbol{H}_{K}\right.$, $\left.\boldsymbol{T}_{1}, \ldots, \boldsymbol{T}_{L}\right)$ of size $n(\leq m)$, are ordinarily related if an $m \times n$ matrix $\boldsymbol{V}$ exists such that

1. $\mathbf{1}_{m}=\boldsymbol{V} \mathbf{1}_{n}$,

2. $\pi V=\phi$,

3. $\boldsymbol{G}_{k} \boldsymbol{V}=\boldsymbol{V} \boldsymbol{H}_{k}, k=0, \ldots, K$, and $\boldsymbol{U}_{l} \boldsymbol{V}=\boldsymbol{V} \boldsymbol{T}_{l}, l=1, \ldots, L$.

Definition 7. Two EMRAPs, $\left(\boldsymbol{\pi}, \boldsymbol{G}_{0}, \ldots, \boldsymbol{G}_{K}, \boldsymbol{U}_{1}, \ldots, \boldsymbol{U}_{L}\right)$ of size $m$ and $\left(\boldsymbol{\phi}, \boldsymbol{H}_{0}, \ldots, \boldsymbol{H}_{K}\right.$, $\left.\boldsymbol{T}_{1}, \ldots, \boldsymbol{T}_{L}\right)$ of size $n(\leq m)$, are weakly related if an $n \times m$ matrix $\boldsymbol{W}$ exists such that

1. $\mathbf{1}_{n}=\boldsymbol{W} \mathbf{1}_{m}$,

2. $\pi=\phi W$,

3. $\boldsymbol{W} \boldsymbol{G}_{k}=\boldsymbol{H}_{k} \boldsymbol{W}, k=0, \ldots, K$, and $\boldsymbol{W} \boldsymbol{U}_{l}=\boldsymbol{T}_{l} \boldsymbol{W}, l=1, \ldots, L$. 
The following two theorems show the preservation of the relations by composition.

Theorem 5. Let $\left(\boldsymbol{\pi}^{(1)}, \boldsymbol{G}_{0}^{(1)}, \ldots, \boldsymbol{G}_{K}^{(1)}, \boldsymbol{U}_{1}^{(1)}, \ldots, \boldsymbol{U}_{L}^{(1)}\right)$ of size $m_{1}$ and $\left(\boldsymbol{\phi}^{(1)}, \boldsymbol{H}_{0}^{(1)}, \ldots, \boldsymbol{H}_{K}^{(1)}\right.$, $\left.\boldsymbol{T}_{1}^{(1)}, \ldots, \boldsymbol{T}_{L}^{(1)}\right)$ of size $n_{1}\left(<m_{1}\right)$ be two EMRAPs that are ordinarily related with matrix $\boldsymbol{V}$, and let $\left(\boldsymbol{\pi}^{(2)}, \boldsymbol{G}_{0}^{(2)}, \ldots, \boldsymbol{G}_{L}^{(2)}, \boldsymbol{U}_{1}^{(2)}, \ldots, \boldsymbol{U}_{K}^{(2)}\right)$ of size $n_{2}$ be another EMRAP. Let $\left(\boldsymbol{\pi}^{(0)}, \boldsymbol{G}_{0}^{(0)}, \ldots\right.$, $\left.\boldsymbol{G}_{K+L}^{(0)}\right)$ and $\left(\boldsymbol{\phi}^{(0)}, \boldsymbol{H}_{0}^{(0)}, \ldots, \boldsymbol{H}_{K+L}^{(0)}\right)$ be the MRAPs resulting from the composition of $\left(\boldsymbol{\pi}^{(1)}\right.$, $\left.\boldsymbol{G}_{0}^{(1)}, \ldots, \boldsymbol{G}_{K}^{(1)}, \boldsymbol{U}_{1}^{(1)}, \ldots, \boldsymbol{U}_{L}^{(1)}\right)$ with $\left(\boldsymbol{\pi}^{(2)}, \boldsymbol{G}_{0}^{(2)}, \ldots, \boldsymbol{G}_{L}^{(2)}, \boldsymbol{U}_{1}^{(2)}, \ldots, \boldsymbol{U}_{K}^{(2)}\right)$ and $\left(\boldsymbol{\phi}^{(1)}, \boldsymbol{H}_{0}^{(1)}\right.$, $\left.\ldots, \boldsymbol{H}_{K}^{(1)}, \boldsymbol{T}_{1}^{(1)}, \ldots, \boldsymbol{T}_{L}^{(1)}\right)$ with $\left(\boldsymbol{\pi}^{(2)}, \boldsymbol{G}_{0}^{(2)}, \ldots, \boldsymbol{G}_{L}^{(2)}, \boldsymbol{U}_{1}^{(2)}, \ldots, \boldsymbol{U}_{K}^{(2)}\right)$, respectively. Then $\left(\boldsymbol{\pi}^{(0)}, \boldsymbol{G}_{0}^{(0)}, \ldots, \boldsymbol{G}_{K+L}^{(0)}\right)$ and $\left(\boldsymbol{\phi}^{(0)}, \boldsymbol{H}_{0}^{(0)}, \ldots, \boldsymbol{H}_{K+L}^{(0)}\right)$ are ordinarily related with matrix $\boldsymbol{V}^{(0)}=$ $\boldsymbol{V} \otimes \boldsymbol{I}_{n_{2}}$.

Proof. We have to prove that the three conditions given in Definition 3 hold. The first holds since $\left(\boldsymbol{V} \otimes \boldsymbol{I}_{n_{2}}\right) \mathbf{1}_{n_{1} n_{2}}=\boldsymbol{V} \mathbf{1}_{n_{1}} \otimes \boldsymbol{I}_{n_{2}} \mathbf{1}_{n_{2}}=\mathbf{1}_{m_{1}} \otimes \mathbf{1}_{n_{2}}=\mathbf{1}_{m_{1} n_{2}}$. Observe that $\boldsymbol{V}^{(0)}$ is an $m_{1} n_{2} \times n_{1} n_{2}$ matrix. For the second condition, we have

$$
\boldsymbol{\pi}^{(0)} \boldsymbol{V}^{(0)}=\left(\boldsymbol{\pi}^{(1)} \otimes \boldsymbol{\pi}^{(2)}\right)\left(\boldsymbol{V} \otimes \boldsymbol{I}_{n_{2}}\right)=\boldsymbol{\pi}^{(1)} \boldsymbol{V} \otimes \boldsymbol{\pi}^{(2)}=\boldsymbol{\phi}^{(1)} \otimes \boldsymbol{\pi}^{(2)}=\boldsymbol{\phi}^{(0)} .
$$

Finally, we have

$$
\begin{aligned}
\boldsymbol{G}_{0}^{(0)} \boldsymbol{V}^{(0)} & =\left(\boldsymbol{G}_{0}^{(1)} \otimes \boldsymbol{I}_{n_{2}}+\boldsymbol{I}_{m_{1}} \otimes \boldsymbol{G}_{0}^{(2)}\right)\left(\boldsymbol{V} \otimes \boldsymbol{I}_{n_{2}}\right) \\
& =\boldsymbol{G}_{0}^{(1)} \boldsymbol{V} \otimes \boldsymbol{I}_{n_{2}}+\boldsymbol{V} \otimes \boldsymbol{G}_{0}^{(2)} \\
& =\boldsymbol{V} \boldsymbol{H}_{0}^{(1)} \otimes \boldsymbol{I}_{n_{2}}+\boldsymbol{V} \otimes \boldsymbol{G}_{0}^{(2)} \\
& =\left(\boldsymbol{V} \otimes \boldsymbol{I}_{n_{2}}\right)\left(\boldsymbol{H}_{0}^{(1)} \otimes \boldsymbol{I}_{n_{2}}+\boldsymbol{I}_{n_{1}} \otimes \boldsymbol{G}_{0}^{(2)}\right) \\
& =\boldsymbol{V}^{(0)} \boldsymbol{H}_{0}^{(0)} \\
\boldsymbol{G}_{k}^{(0)} \boldsymbol{V}^{(0)} & =\left(\boldsymbol{G}_{k}^{(1)} \otimes \boldsymbol{U}_{k}^{(2)}\right)\left(\boldsymbol{V} \otimes \boldsymbol{I}_{n_{2}}\right) \\
& =\boldsymbol{G}_{k}^{(1)} \boldsymbol{V} \otimes \boldsymbol{U}_{k}^{(2)} \\
& =\boldsymbol{V} \boldsymbol{H}_{k}^{(1)} \otimes \boldsymbol{U}_{k}^{(2)} \\
& =\boldsymbol{V}^{(0)} \boldsymbol{G}_{k}^{(0)} \quad \text { if } 1 \leq k \leq K, \\
\boldsymbol{G}_{k}^{(0)} \boldsymbol{V}^{(0)} & =\left(\boldsymbol{U}_{k-K}^{(1)} \otimes \boldsymbol{G}_{k-K}^{(2)}\right)\left(\boldsymbol{V} \otimes \boldsymbol{I}_{n_{2}}\right) \\
& =\boldsymbol{U}_{k-K}^{(1)} \boldsymbol{V} \otimes \boldsymbol{G}_{k-K}^{(2)} \\
& =\boldsymbol{V} \boldsymbol{T}_{k-K}^{(1)} \otimes \boldsymbol{G}_{k-K}^{(2)} \\
& =\boldsymbol{V}^{(0)} \boldsymbol{G}_{k}^{(0)} \quad \text { if } K<k \leq K+L .
\end{aligned}
$$

It is easy to show that the relation also holds if we exchange the indices 1 and 2 . In this case, $\boldsymbol{V}^{(0)}=\boldsymbol{I} \otimes \boldsymbol{V}$.

Theorem 6. Let $\left(\boldsymbol{\pi}^{(1)}, \boldsymbol{G}_{0}^{(1)}, \ldots, \boldsymbol{G}_{K}^{(1)}, \boldsymbol{U}_{1}^{(1)}, \ldots, \boldsymbol{U}_{L}^{(1)}\right)$ of size $m_{1}$ and $\left(\boldsymbol{\phi}^{(1)}, \boldsymbol{H}_{0}^{(1)}, \ldots, \boldsymbol{H}_{K}^{(1)}\right.$, $\left.\boldsymbol{T}_{1}^{(1)}, \ldots, \boldsymbol{T}_{L}^{(1)}\right)$ of size $n_{1}\left(<m_{1}\right)$ be two EMRAPs that are weakly related with matrix $\boldsymbol{W}$, and let $\left(\boldsymbol{\pi}^{(2)}, \boldsymbol{G}_{0}^{(2)}, \ldots, \boldsymbol{G}_{L}^{(2)}, \boldsymbol{U}_{1}^{(2)}, \ldots, \boldsymbol{U}_{K}^{(2)}\right)$ of size $n_{2}$ be another EMRAP. Let $\left(\boldsymbol{\pi}^{(0)}, \boldsymbol{G}_{0}^{(0)}, \ldots\right.$, $\left.\boldsymbol{G}_{K+L}^{(0)}\right)$ and $\left(\boldsymbol{\phi}^{(0)}, \boldsymbol{H}_{0}^{(0)}, \ldots, \boldsymbol{H}_{K+L}^{(0)}\right)$ be the MRAPs resulting from the composition of $\left(\boldsymbol{\pi}^{(1)}\right.$, $\left.\boldsymbol{G}_{0}^{(1)}, \ldots, \boldsymbol{G}_{K}^{(1)}, \boldsymbol{U}_{1}^{(1)}, \ldots, \boldsymbol{U}_{L}^{(1)}\right)$ with $\left(\boldsymbol{\pi}^{(2)}, \boldsymbol{G}_{0}^{(2)}, \ldots, \boldsymbol{G}_{L}^{(2)}, \boldsymbol{U}_{1}^{(2)}, \ldots, \boldsymbol{U}_{K}^{(2)}\right)$ and $\left(\boldsymbol{\phi}^{(1)}, \boldsymbol{H}_{0}^{(1)}\right.$, $\left.\ldots, \boldsymbol{H}_{K}^{(1)}, \boldsymbol{T}_{1}^{(1)}, \ldots, \boldsymbol{T}_{L}^{(1)}\right)$ with $\left(\boldsymbol{\pi}^{(2)}, \boldsymbol{G}_{0}^{(2)}, \ldots, \boldsymbol{G}_{L}^{(2)}, \boldsymbol{U}_{1}^{(2)}, \ldots, \boldsymbol{U}_{K}^{(2)}\right)$, respectively. Then $\left(\boldsymbol{\pi}^{(0)}, \boldsymbol{G}_{0}^{(0)}, \ldots, \boldsymbol{G}_{K+L}^{(0)}\right)$ and $\left(\boldsymbol{\phi}^{(0)}, \boldsymbol{H}_{0}^{(0)}, \ldots, \boldsymbol{H}_{K+L}^{(0)}\right)$ are weakly related with matrix $\boldsymbol{W}^{(0)}=$ $\boldsymbol{W} \otimes \boldsymbol{I}_{n_{2}}$. 
Proof. The proof follows the proof of Theorem 5.

Again, the result holds if we exchange the indices 1 and 2 such that the composed processes are weakly related with $\boldsymbol{W}^{(0)}=\boldsymbol{I} \otimes \boldsymbol{W}$.

If the representations are ordinarily or weakly related they are equivalent processes and can be substituted without changing the joint densities. The following corollary combines the results of the previous theorems and shows that the result allows compositional modeling by first finding smaller ordinarily/weakly related representations which are then composed with other processes, resulting in a valid MRAP.

Corollary 3. Let $\left(\boldsymbol{\pi}^{(1)}, \boldsymbol{G}_{0}^{(1)}, \ldots, \boldsymbol{G}_{K}^{(1)}, \boldsymbol{U}_{1}^{(1)}, \ldots, \boldsymbol{U}_{L}^{(1)}\right)$ of size $m_{1}$ and $\left(\boldsymbol{\phi}^{(1)}, \boldsymbol{H}_{0}^{(1)}, \ldots, \boldsymbol{H}_{K}^{(1)}\right.$, $\left.\boldsymbol{T}_{1}^{(1)}, \ldots, \boldsymbol{T}_{L}^{(1)}\right)$ of size $n_{1}\left(<m_{1}\right)$ be two EMRAPs that are ordinarily or weakly related. Let $\left(\boldsymbol{\pi}^{(2)}, \boldsymbol{G}_{0}^{(2)}, \ldots, \boldsymbol{G}_{L}^{(2)}, \boldsymbol{U}_{1}^{(2)}, \ldots, \boldsymbol{U}_{K}^{(2)}\right)$ of size $m_{2}$ and $\left(\boldsymbol{\phi}^{(2)}, \boldsymbol{H}_{0}^{(2)}, \ldots, \boldsymbol{H}_{L}^{(2)}, \boldsymbol{T}_{1}^{(2)}, \ldots, \boldsymbol{T}_{K}^{(2)}\right)$ of size $n_{2}\left(\leq m_{2}\right)$ be two EMRAPs which are ordinarily or weakly related. Let $\left(\boldsymbol{\pi}^{(0)}, \boldsymbol{G}_{0}^{(0)}, \ldots\right.$, $\left.\boldsymbol{G}_{K+L}^{(0)}\right)$ be the MRAP resulting from the composition of $\left(\boldsymbol{\pi}^{(1)}, \boldsymbol{G}_{0}^{(1)}, \ldots, \boldsymbol{G}_{K}^{(1)}, \boldsymbol{U}_{1}^{(1)}, \ldots, \boldsymbol{U}_{L}^{(1)}\right)$ and $\left(\boldsymbol{\pi}^{(2)}, \boldsymbol{G}_{0}^{(2)}, \ldots, \boldsymbol{G}_{L}^{(2)}, \boldsymbol{U}_{1}^{(2)}, \ldots, \boldsymbol{U}_{K}^{(2)}\right)$, and let $\left(\boldsymbol{\phi}^{(0)}, \boldsymbol{H}_{0}^{(0)}, \ldots, \boldsymbol{H}_{K+L}^{(0)}\right)$ be the MRAP resulting from the composition of $\left(\boldsymbol{\phi}^{(1)}, \boldsymbol{H}_{0}^{(1)}, \ldots, \boldsymbol{H}_{K}^{(1)}, \boldsymbol{T}_{1}^{(1)}, \ldots, \boldsymbol{T}_{L}^{(1)}\right)$ and $\left(\boldsymbol{\phi}^{(2)}, \boldsymbol{H}_{0}^{(2)}, \ldots\right.$, $\left.\boldsymbol{H}_{L}^{(2)}, \boldsymbol{T}_{1}^{(2)}, \ldots, \boldsymbol{T}_{K}^{(2)}\right)$. Then $\left(\boldsymbol{\pi}^{(0)}, \boldsymbol{G}_{0}^{(0)}, \ldots, \boldsymbol{G}_{K+L}^{(0)}\right)$ and $\left(\boldsymbol{\phi}^{(0)}, \boldsymbol{H}_{0}^{(0)}, \ldots, \boldsymbol{H}_{K+L}^{(0)}\right)$ are equivalent.

Proof. We assume that $\left(\boldsymbol{\pi}^{(1)}, \boldsymbol{G}_{0}^{(1)}, \ldots, \boldsymbol{G}_{K}^{(1)}, \boldsymbol{U}_{1}^{(1)}, \ldots, \boldsymbol{U}_{L}^{(1)}\right)$ and $\left(\boldsymbol{\phi}^{(1)}, \boldsymbol{H}_{0}^{(1)}, \ldots, \boldsymbol{H}_{K}^{(1)}\right.$, $\left.\boldsymbol{T}_{1}^{(1)}, \ldots, \boldsymbol{T}_{L}^{(1)}\right)$ are ordinarily related, and that $\left(\boldsymbol{\pi}^{(2)}, \boldsymbol{G}_{0}^{(2)}, \ldots, \boldsymbol{G}_{L}^{(2)}, \boldsymbol{U}_{1}^{(2)}, \ldots, \boldsymbol{U}_{K}^{(2)}\right)$ and $\left(\boldsymbol{\phi}^{(2)}\right.$, $\left.\boldsymbol{H}_{0}^{(2)}, \ldots, \boldsymbol{H}_{L}^{(2)}, \boldsymbol{T}_{1}^{(2)}, \ldots, \boldsymbol{T}_{K}^{(2)}\right)$ are weakly related. The other cases are proved similarly.

First, we consider the EMRAP $\left(\boldsymbol{\eta}^{(0)}, \boldsymbol{F}_{0}^{(0)}, \ldots, \mathbf{F}_{(K+L}^{(0)}\right)$ which results from the composition of $\left(\boldsymbol{\phi}^{(1)}, \boldsymbol{H}_{0}^{(1)}, \ldots, \boldsymbol{H}_{K}^{(1)}, \boldsymbol{T}_{1}^{(1)}, \ldots, \boldsymbol{T}_{L}^{(1)}\right)$ and $\left(\boldsymbol{\pi}^{(2)}, \boldsymbol{G}_{0}^{(2)}, \ldots, \boldsymbol{G}_{L}^{(2)}, \boldsymbol{U}_{1}^{(2)}, \ldots, \boldsymbol{U}_{K}^{(2)}\right)$. According to Theorem 5, this MRAP is equivalent to $\left(\boldsymbol{\pi}^{(0)}, \boldsymbol{G}_{0}^{(0)}, \ldots, \boldsymbol{G}_{K+L}^{(0)}\right)$.

Then we can start with $\left(\boldsymbol{\eta}^{(0)}, \boldsymbol{F}_{0}^{(0)}, \ldots, \mathbf{F}_{(K+L}^{(0)}\right)$ and, according to Theorem 6 , this EMRAP is equivalent to $\left(\boldsymbol{\phi}^{(0)}, \boldsymbol{H}_{0}^{(0)}, \ldots, \boldsymbol{H}_{K+L}^{(0)}\right)$. Since equivalence of MRAPs is transitive, the corollary follows.

Example 3. We consider the following two EMRAPs which are both Markovian:

$$
\begin{aligned}
& \boldsymbol{\pi}^{(1)}=(0.563484,0.380168,0.012697,0.043652), \\
& \boldsymbol{G}_{0}^{(1)}=\left(\begin{array}{cccc}
-1 & 1 & 0 & 0 \\
0 & -1 & 1 & 0 \\
0 & 0 & -1 & 1 \\
0 & 0 & 0 & -2.290867
\end{array}\right) \text {, } \\
& \boldsymbol{G}_{1}^{(1)}=\left(\begin{array}{cccc}
0 & 0 & 0 & 0 \\
0 & 0 & 0 & 0 \\
0 & 0 & 0 & 0 \\
1.290867 & 1 & 0 & 0
\end{array}\right) \\
& \boldsymbol{U}_{1}^{(1)}=\left(\begin{array}{cccc}
0.825445 & 0.174555 & 0 & 0 \\
0.225327 & 0.774673 & 0 & 0 \\
1 & 0 & 0 & 0 \\
0 & 1 & 0 & 0
\end{array}\right) \text {, }
\end{aligned}
$$


and

$$
\begin{aligned}
& \boldsymbol{\pi}^{(2)}=(0.1,0.9,0,0), \\
& \boldsymbol{G}_{0}^{(2)}=\left(\begin{array}{cccc}
-0.83556 & 0.42667 & 0 & 0.08889 \\
0 & -0.9775 & 0.67 & 0.2475 \\
0.40222 & 1.17333 & -2 & 0.24444 \\
0.29111 & 1.30667 & 0 & -1.97778
\end{array}\right), \\
& \boldsymbol{G}_{1}^{(2)}=\left(\begin{array}{cccc}
0.03556 & 0.19833 & 0 & 0.08611 \\
0.00667 & 0.045 & 0 & 0.00833 \\
0.02 & 0.1225 & 0 & 0.0375 \\
0.04222 & 0.26833 & 0 & 0.06944
\end{array}\right) \\
& \boldsymbol{U}_{1}^{(2)}=\left(\begin{array}{cccc}
0 & 0.475 & 0 & 0.525 \\
0.61111 & 0.09167 & 0 & 0.29722 \\
0.38889 & 0.18333 & 0 & 0.42778 \\
0.16667 & 0.125 & 0 & 0.70833
\end{array}\right)
\end{aligned}
$$

Composition of the two processes results in an MMAP $\left(\boldsymbol{\pi}^{(0)}, \boldsymbol{G}_{0}^{(0)}, \boldsymbol{G}_{1}^{(0)}, \boldsymbol{G}_{2}^{(0)}\right)$ with 16 states. However, the first EMRAP is weakly related with matrix

$$
\boldsymbol{W}=\left(\begin{array}{cccc}
0.563484 & 0.436516 & 0 & 0 \\
0 & 0.563484 & 0.436516 & 0 \\
0 & 0 & 0.563484 & 0.436516
\end{array}\right)
$$

to the EMRAP

$$
\begin{array}{ccc}
\boldsymbol{\phi}^{(1)}=(1,-0.1,0.1), & \boldsymbol{H}_{0}^{(1)}=\left(\begin{array}{ccc}
-1 & 1 & 0 \\
0 & -1 & 1 \\
0 & 0 & -1
\end{array}\right), \\
\boldsymbol{H}_{1}^{(1)}=\left(\begin{array}{lll}
0 & 0 & 0 \\
0 & 0 & 0 \\
1 & 0 & 0
\end{array}\right), & \boldsymbol{T}_{1}^{(1)}=\left(\begin{array}{lll}
1 & 0 & 0 \\
1 & 0 & 0 \\
1 & 0 & 0
\end{array}\right) .
\end{array}
$$

The second EMRAP is ordinarily related with matrix

$$
\boldsymbol{V}=\left(\begin{array}{ccc}
0.8 & 0.4 & -0.2 \\
-0.1 & 0.2 & 0.9 \\
0.1 & 0.4 & 0.5 \\
-0.1 & 1 & 0.1
\end{array}\right)
$$

to the EMRAP

$$
\begin{array}{ccc}
\boldsymbol{\phi}^{(2)}=(-0.01,0.22,0.79), & \boldsymbol{H}_{0}^{(2)}=\left(\begin{array}{ccc}
-1 & 0.8 & 0 \\
0.2 & -1.6 & 1 \\
0 & 0.8 & -0.8
\end{array}\right), \\
\boldsymbol{H}_{1}^{(2)}=\left(\begin{array}{ccc}
0 & 0.1 & 0.1 \\
0 & 0.15 & 0.25 \\
0 & 0 & 0
\end{array}\right), & \boldsymbol{T}_{1}^{(2)}=\left(\begin{array}{ccc}
0 & 0.5 & 0.5 \\
0 & 0.8 & 0.2 \\
0.5 & 0.5 & 0
\end{array}\right) .
\end{array}
$$

It is interesting to note that in both cases only the initial vector prohibits the processes being Markovian. Composition of the two processes results in an MRAP $\left(\boldsymbol{\phi}^{(0)}, \boldsymbol{H}_{0}^{(0)}, \boldsymbol{H}_{1}^{(0)}, \boldsymbol{H}_{2}^{(2)}\right)$ 
with nine states characterized by the following vector and matrices:

$$
\begin{aligned}
& \boldsymbol{\phi}^{(0)}=(0.01,0.22,0.79,0.001,-0.022,-0.079,-0.001,0.022,0.079) \text {, } \\
& \boldsymbol{H}_{0}^{(0)}=\left(\begin{array}{ccccccccc}
-2 & 0.8 & 0 & 1 & 0 & 0 & 0 & 0 & 0 \\
0.2 & -2.6 & 1 & 0 & 1 & 0 & 0 & 0 & 0 \\
0 & 0.8 & -1.8 & 0 & 0 & 1 & 0 & 0 & 0 \\
0 & 0 & 0 & -2 & 0.8 & 0 & 1 & 0 & 0 \\
0 & 0 & 0 & 0.2 & -2.6 & 1 & 0 & 1 & 0 \\
0 & 0 & 0 & 0 & 0.8 & -1.8 & 0 & 0 & 1 \\
0 & 0 & 0 & 0 & 0 & 0 & -2 & 0.8 & 0 \\
0 & 0 & 0 & 0 & 0 & 0 & 0.2 & -2.6 & 1 \\
0 & 0 & 0 & 0 & 0 & 0 & 0 & 0.8 & -1.8
\end{array}\right), \\
& \boldsymbol{H}_{1}^{(0)}=\left(\begin{array}{ccccccccc}
0 & 0 & 0 & 0 & 0 & 0 & 0 & 0 & 0 \\
0 & 0 & 0 & 0 & 0 & 0 & 0 & 0 & 0 \\
0 & 0 & 0 & 0 & 0 & 0 & 0 & 0 & 0 \\
0 & 0 & 0 & 0 & 0 & 0 & 0 & 0 & 0 \\
0 & 0 & 0 & 0 & 0 & 0 & 0 & 0 & 0 \\
0 & 0 & 0 & 0 & 0 & 0 & 0 & 0 & 0 \\
0 & 0.5 & 0.5 & 0 & 0 & 0 & 0 & 0 & 0 \\
0 & 0.8 & 0.2 & 0 & 0 & 0 & 0 & 0 & 0 \\
0 & 0.5 & 0 & 0 & 0 & 0 & 0 & 0 & 0
\end{array}\right) \\
& \boldsymbol{H}_{2}^{(0)}=\left(\begin{array}{ccccccccc}
0 & 0.1 & 0.1 & 0 & 0 & 0 & 0 & 0 & 0 \\
0 & 0.15 & 0.25 & 0 & 0 & 0 & 0 & 0 & 0 \\
0 & 0 & 0 & 0 & 0 & 0 & 0 & 0 & 0 \\
0 & 0.1 & 0.1 & 0 & 0 & 0 & 0 & 0 & 0 \\
0 & 0.15 & 0.25 & 0 & 0 & 0 & 0 & 0 & 0 \\
0 & 0 & 0 & 0 & 0 & 0 & 0 & 0 & 0 \\
0 & 0.1 & 0.1 & 0 & 0 & 0 & 0 & 0 & 0 \\
0 & 0.15 & 0.25 & 0 & 0 & 0 & 0 & 0 & 0 \\
0 & 0 & 0 & 0 & 0 & 0 & 0 & 0 & 0
\end{array}\right)
\end{aligned}
$$

Both MRAPs $\left(\boldsymbol{\pi}^{(0)}, \boldsymbol{G}_{0}^{(0)}, \boldsymbol{G}_{1}^{(0)}, \boldsymbol{G}_{2}^{(0)}\right)$ and $\left(\boldsymbol{\phi}^{(0)}, \boldsymbol{H}_{0}^{(0)}, \boldsymbol{H}_{1}^{(0)}, \boldsymbol{H}_{2}^{(0)}\right)$ are equivalent. The former MRAP is an MMAP, the latter is not an MMAP since the initial vector contains negative elements. The matrices are in both cases MMAP matrices. Furthermore, $\left(\boldsymbol{G}_{0}\right)^{-1} \sum_{k=1}^{K} \boldsymbol{G}_{k}$ and $\left(\boldsymbol{H}_{0}\right)^{-1} \sum_{k=1}^{K} \boldsymbol{H}_{k}$ are irreducible stochastic matrices such that the embedded stationary vector can in both cases be computed as the left eigenvector belonging to the unique eigenvalue 1 of the above matrices. The resulting eigenvectors normalized to 1 describe a probability distribution. This implies starting from the embedded stationary vector both MRAPs are MMAPs. However, the initial vector $\boldsymbol{\phi}^{(0)}$ determined by $\boldsymbol{\pi}^{(0)}$ is not a proper distribution.

\section{Conclusions and future work}

In this paper we defined a new class of stochastic processes, called marked rational arrival processes (MRAPs), which are a natural extension of rational arrival processes (RAPs). Furthermore, we introduced two equivalence relations for these processes which are generalizations of ordinary and weak lumpability defined for Markov processes. We showed that the equivalence relations allow us to relate Markovian and non-Markovian representations, and that 
the equivalence is preserved by asynchronous composition of MRAPs, which is also defined in the paper.

The class of MRAPs offers interesting possibilities in stochastic modeling since processes can be analyzed numerically even if they are not Markovian. MRAPs with finite state spaces are more general than Markov models with finite state spaces, however, a complete characterization of the relation between MRAPs and MMAPs is still missing. Additionally, the development of algorithms to compute the proposed relations between different MRAPs is also an important point. A first approach can be found in [10].

\section{Appendix A. Ordinary relation with matrix $V$ of reduced column rank}

Theorem 7. Assume that MRAPs $\left(\boldsymbol{\pi}, \boldsymbol{G}_{0}, \ldots, \boldsymbol{G}_{K}\right)$ of size $m$ and $\left(\boldsymbol{\phi}, \boldsymbol{H}_{0}, \ldots, \boldsymbol{H}_{K}\right)$ of size $n$ $(n \leq m)$ are related as in Definition 3, but that $\operatorname{rank}(\boldsymbol{V})=r<n$. Then there exists an $m \times r$ matrix $\boldsymbol{U}$ and an $\operatorname{MRAP}\left(\boldsymbol{\eta}, \boldsymbol{F}_{0}, \ldots, \boldsymbol{F}_{K}\right)$ of size $r$ such that $\left(\boldsymbol{\pi}, \boldsymbol{G}_{0}, \ldots, \boldsymbol{G}_{K}\right)$ and $\left(\boldsymbol{\eta}, \boldsymbol{F}_{0}, \ldots, \boldsymbol{F}_{K}\right)$ are ordinarily related by matrix $\boldsymbol{U}$.

Proof. We show how to compute the matrix $\boldsymbol{U}$ and MRAP $\left(\boldsymbol{\eta}, \boldsymbol{F}_{0}, \ldots, \boldsymbol{F}_{K}\right)$. Without loss of generality, we assume that the first $r$ columns of $\boldsymbol{V}$ are linearly independent such that $\boldsymbol{V}=\left(\boldsymbol{V}_{1} \boldsymbol{V}_{2}\right)$ and $\boldsymbol{V}_{1}$ is an $m \times r$ matrix with $\operatorname{rank}\left(\boldsymbol{V}_{1}\right)=r$. Since the columns of $\boldsymbol{V}_{1}$ spawn the column space of $\boldsymbol{V}$, an $r \times s(s=n-r)$ matrix $\boldsymbol{A}$ exists and $\boldsymbol{V}_{2}=\boldsymbol{V}_{1} \boldsymbol{A}$. Let

$$
\boldsymbol{H}_{k}=\left(\begin{array}{cc}
\boldsymbol{H}_{k}^{1} & \boldsymbol{H}_{k}^{2} \\
\boldsymbol{H}_{k}^{3} & \boldsymbol{H}_{k}^{4}
\end{array}\right),
$$

where $\boldsymbol{H}_{k}^{1}$ is an $r \times r$ matrix. Here $\boldsymbol{G}_{k} \boldsymbol{V}=\boldsymbol{V} \boldsymbol{H}_{k}$ implies that $\boldsymbol{G}_{k} \boldsymbol{V}_{1}=\boldsymbol{V}_{1}\left(\boldsymbol{H}_{k}^{1}+\boldsymbol{A} \boldsymbol{H}_{k}^{3}\right)$. Define $\boldsymbol{D}=\boldsymbol{I}_{r}+\operatorname{diag}\left(\boldsymbol{A \mathbf { 1 } _ { s }}\right)$, an $r \times r$ matrix, where $\operatorname{diag}(\cdot)$ of a vector is a diagonal matrix with the vector elements in the diagonal. If $\boldsymbol{U}=\boldsymbol{V}_{1} \boldsymbol{D}$ then

$$
\boldsymbol{U} \mathbf{1}_{r}=\boldsymbol{V}_{1} D \mathbf{1}_{r}=\boldsymbol{V}_{1}\left(\boldsymbol{I}_{r}+\operatorname{diag}\left(\boldsymbol{A} \mathbf{1}_{s}\right)\right) \mathbf{1}_{r}=\boldsymbol{V}_{1}\left(\boldsymbol{I}_{r} \mathbf{1}_{r}+\boldsymbol{A} \mathbf{1}_{s}\right)=\boldsymbol{V}_{1} \mathbf{1}_{r}+\boldsymbol{V}_{2} \mathbf{1}_{s}=\mathbf{1}_{n}
$$

The relation also implies that $\boldsymbol{D}$ cannot be $\mathbf{0}$ but may contain 0 diagonal elements. We first assume that $\boldsymbol{D}$ is nonsingular, i.e. all diagonal elements are nonzero. Define the vector $\boldsymbol{\eta}=\boldsymbol{\pi} \boldsymbol{U}$ and the matrices $\boldsymbol{F}_{k}=\boldsymbol{D}^{-1}\left(\boldsymbol{H}_{k}^{1}+\boldsymbol{A} \boldsymbol{H}_{k}^{3}\right) \boldsymbol{D}$ such that

$$
\boldsymbol{U} \boldsymbol{F}_{k}=\boldsymbol{V}_{1} \boldsymbol{D} \boldsymbol{D}^{-1}\left(\boldsymbol{H}_{k}^{1}+\boldsymbol{A} \boldsymbol{H}_{k}^{3}\right) \boldsymbol{D}=\boldsymbol{V}_{1}\left(\boldsymbol{H}_{k}^{1}+\boldsymbol{A} \boldsymbol{H}_{k}^{3}\right) \boldsymbol{D}=\boldsymbol{G}_{k} \boldsymbol{V}_{1} \boldsymbol{D}=\boldsymbol{G}_{k} \boldsymbol{U} .
$$

Since $\boldsymbol{D}$ has full rank, $\operatorname{rank}\left(\boldsymbol{V}_{1} \boldsymbol{D}\right)=\operatorname{rank}\left(\boldsymbol{V}_{1}\right)=r$, which completes the proof for this case.

If $\boldsymbol{D}$ is singular, we assume without loss of generality that the first $u>0$ diagonal elements are nonzero such that

$$
\boldsymbol{D}=\left(\begin{array}{cc}
\boldsymbol{D}_{1} & \mathbf{0} \\
\mathbf{0} & \mathbf{0}
\end{array}\right),
$$

where $\boldsymbol{D}_{1}$, of size $u \times u$, is a nonsingular diagonal matrix. We define

$$
\tilde{\boldsymbol{D}}=\left(\begin{array}{cc}
\boldsymbol{D}_{1} & \mathbf{0} \\
-\mathbf{1}_{v} \boldsymbol{e}_{0} & \boldsymbol{I}_{v}
\end{array}\right)
$$

Then

$$
\tilde{\boldsymbol{D}}^{-1}=\left(\begin{array}{cc}
\boldsymbol{D}_{1}^{-1} & \mathbf{0} \\
\left(\mathbf{1}_{v} \boldsymbol{e}_{0}\right) \boldsymbol{D}_{1}^{-1} & \boldsymbol{I}_{v}
\end{array}\right),
$$


where $v=r-u, \boldsymbol{I}_{v}$ is the identity matrix of order $v$, and $\boldsymbol{e}_{0}$ is a row vector of length $u$ (whose elements are numbered from 0 to $u-1$ ). The element 0 of $\boldsymbol{e}_{0}$ is 1 and all other elements are 0 . Define $\tilde{\boldsymbol{U}}=\boldsymbol{V}_{1} \tilde{\boldsymbol{D}}$. Since $\boldsymbol{D} \mathbf{1}_{r}=\tilde{\boldsymbol{D}} \mathbf{1}_{r}$, we have $\tilde{\boldsymbol{U}} \mathbf{1}_{r}=\mathbf{1}_{r}$. Now we can use $\tilde{\boldsymbol{D}}$ and $\tilde{\boldsymbol{U}}$ instead of $\boldsymbol{D}$ and $\boldsymbol{U}$ and obtain, for $\boldsymbol{F}_{k}=\tilde{\boldsymbol{D}}^{-1}\left(\boldsymbol{H}_{k}^{1}+\boldsymbol{A} \boldsymbol{H}_{k}^{3}\right) \tilde{\boldsymbol{D}}$,

$$
\tilde{\boldsymbol{U}} \boldsymbol{F}_{k}=\boldsymbol{V}_{1} \tilde{\boldsymbol{D}} \tilde{\boldsymbol{D}}^{-1}\left(\boldsymbol{H}_{k}^{1}+\boldsymbol{A} \boldsymbol{H}_{k}^{3}\right) \tilde{\boldsymbol{D}}=\boldsymbol{V}_{1}\left(\boldsymbol{H}_{k}^{1}+\boldsymbol{A} \boldsymbol{H}_{k}^{3}\right) \tilde{\boldsymbol{D}}=\boldsymbol{G}_{k} \boldsymbol{V}_{1} \tilde{\boldsymbol{D}}=\boldsymbol{G}_{k} \tilde{\boldsymbol{U}}
$$

which completes the proof.

\section{Appendix B. Weak relation with matrix $W$ of reduced row rank}

Theorem 8. Assume that the MRAPs $\left(\boldsymbol{\pi}, \boldsymbol{G}_{0}, \ldots, \boldsymbol{G}_{K}\right)$ of size $m$ and $\left(\boldsymbol{\phi}, \boldsymbol{H}_{0}, \ldots, \boldsymbol{H}_{K}\right)$ of size $n(n \leq m)$ are related as in Definition 5, but that $\operatorname{rank}(\boldsymbol{W})=r<n$. Then there exists an $r \times m$ matrix $\boldsymbol{T}$ and an $\operatorname{MRAP}\left(\boldsymbol{\eta}, \boldsymbol{F}_{0}, \ldots, \boldsymbol{F}_{K}\right)$ of size $r$ such that $\left(\boldsymbol{\pi}, \boldsymbol{G}_{0}, \ldots, \boldsymbol{G}_{K}\right)$ and $\left(\boldsymbol{\eta}, \boldsymbol{F}_{0}, \ldots, \boldsymbol{F}_{K}\right)$ are weakly related by matrix $\boldsymbol{T}$.

Proof. The proof follows the same lines as the proof of Theorem 7.

\section{References}

[1] Akar, N. (2006). Solving the ME/ME/1 queue with state-space methods and the matrix sign function. Performance Evaluation 63, 131-145.

[2] Asmussen, S. And Bladt, M. (1999). Point processes with finite-dimensional conditional probabilities. Stoch. Process. Appl. 82, 127-142.

[3] Asmussen, S. and O'Cinneide, C. A. (1997). Matrix-exponential distributions-distributions with a rational Laplace transform. In Encyclopedia of Statistical Sciences, eds S. Kotz and C. B. Read, John Wiley, New York, pp. 435-440.

[4] Bean, N. G. ANd Nielsen, B. F. (2010). Quasi-birth-and-death processes with rational arrival process components. Stoch. Models 26, 309-334.

[5] Buchiolz, P. (1994). A class of hierarchical queueing networks and their analysis. Queueing Systems $\mathbf{1 5}$, $59-80$.

[6] Buchiolz, P. (1994). Exact and ordinary lumpability in finite Markov chains. J. Appl. Prob. 31, 59-80.

[7] Buchнolz, P. (1995). Equivalence relations for stochastic automata networks. In Computations with Markov Chains, ed. W. J. Stewart, Kluwer, pp. 197-216.

[8] Buchiolz, P. (2008). Bisimulation relations for weighted automata. Theoret. Comput. Sci. 393, 109-123.

[9] Buchiolz, P. and Telek, M. (2010). Stochastic Petri nets with matrix exponentially distributed firing times. Performance Evaluation 67, 1373-1385.

[10] Buchholz, P. and Telek, M. (2011). On minimal representations of rational arrival processes. Ann. Operat. Res. 24 pp.

[11] De Schutter, B. (2000). Minimal state-space realization in linear system theory: an overview. J. Comput. Appl. Math. 121, 331-354.

[12] FaCKrell, M. (2005). Fitting with matrix-exponential distributions. Stoch. Models 21, 377-400.

[13] He, Q.-M. And Neuts, M. F. (1998). Markov chains with marked transitions. Stoch. Process. Appl. 74, $37-52$.

[14] He, Q.-M. and Zhang, H. (2007). On matrix exponential distributions. Adv. Appl. Prob. 39, 271-292.

[15] Hermanns, H. (2002). Interactive Markov Chains. (Lecture Notes Comput. Sci. 2428). Springer, Berlin.

[16] Hillston, J. (1995). Compositional Markovian modelling using a process algebra. In Computations with Markov Chains, ed. W. J. Stewart, Kluwer, pp. 177-196.

[17] Horváth, A., Rácz, S. And Telek, M. (2009). Moments characterization of the class of order 3 matrix exponential distributions. In 16th Internat. Conf. on Analytical and Stochastic Modeling Techniques and Applications (Lecture Notes Comput. Sci. 5513), Springer, Berlin, pp. 174-188.

[18] Kemeny, J. G. And Snell, J. L. (1976). Finite Markov Chains. Springer, New York.

[19] LIPSKY, L. (2008). Queueing Theory. Springer, New York.

[20] Mitchell, K. (2001). Constructing a correlated sequence of matrix exponentials with invariant first-order properties. Operat. Res. Lett. 28, 27-34.

[21] Neuts, M. F. (1979). A versatile Markovian point process. J. Appl. Prob. 16, 764-779.

[22] PARK, D. (1981). Concurrency and automata on infinite sequences. In Theoretical Computer Science (Lecture Notes Comput. Sci. 104), Springer, pp. 167-183. 
[23] Rubino, G. and Sericola, B. (1993). A finite characterization of weak lumpable Markov processes. II. The continuous time case. Stoch. Process. Appl. 45, 115-125.

[24] Stewart, W. J. (1994). Introduction to the Numerical Solution of Markov Chains. Princeton University Press.

[25] Telek, M. And Horváth, G. (2007). A minimal representation of Markov arrival processes and a moments matching method. Performance Evaluation 64, 1153-1168.

[26] Wu, S.-H., Smolka, S. A. And Stark, E. W. (1997). Composition and behaviors of probabilistic I/O automata. Theoret. Comput. Sci. 176, 1-38. 